\title{
Updated pulsation models for anomalous Cepheids
}

\author{
M. Marconi ${ }^{1}$, G. Fiorentino ${ }^{2,3}$, and F. Caputo ${ }^{3}$ \\ 1 Osservatorio Astronomico di Capodimonte, Via Moiariello 16, 80131 Napoli, Italy \\ 2 Universita' Tor Vergata, via della Ricerca Scientifica 1, 00133 Roma, Italy \\ e-mail: giuliana@mporzio.astro.it \\ 3 Osservatorio Astronomico di Roma, Via di Frascati 33, 00040 Monteporzio Catone, Italy \\ e-mail: caputo@coma.mporzio.astro.it
}

Received 11 August 2003 / Accepted 14 January 2004

\begin{abstract}
A theoretical investigation of the pulsation behavior of so-named "anomalous" Cepheids is presented. The study is based on nonlinear convective pulsation models with $Z=0.0001$ and 0.0004 , mass in the range 1.3-2.2 $M_{\odot}$ and various luminosity levels. Based on these computations, we derive periods, bolometric light curves and the edges of the instability strip, showing that a variation of the metal abundance from $Z=0.0001$ to 0.0004 has quite small effects on these quantities. Then, using bolometric corrections and color-temperature transformations, we are able to provide the predicted relations connecting pulsational properties (periods, amplitudes) with magnitudes and colors in the various photometric bands. The theoretical pulsational scenario is compared to observed anomalous Cepheids in dwarf spheroidal galaxies and, in particular, the predicted mass-dependent Period-Magnitude-Amplitude and Period-Magnitude-Color relations are used to estimate individual mass values, as well as to discriminate between fundamental $(\mathrm{F})$ and first-overtone (FO) pulsators.
\end{abstract}

Key words. cepheids - stars: evolution - stars: oscillations

\section{Introduction}

Among the various types of radial pulsating stars, the so-named "anomalous" Cepheids (ACs) are, perhaps, the least explored ones. They are rare in Galactic Globular Clusters, whereas they have been observed in all the nearby dwarf spheroidal galaxies (see e.g., Nemec et al. 1988, 1994; Mateo 1998; Pritzl et al. 2002; Dall'Ora et al. 2003; Baldacci et al. 2003) in which they have been searched for. These variables are intrinsically brighter than RR Lyrae stars and, when their apparent magnitude is scaled to the magnitude level of RR Lyrae stars observed in the same system, they arrange along a strip with the less luminous ones ( $\sim 0.5$ mag brighter than RR Lyrae stars) at periods $\sim 0.30$ days and the more luminous ones $(\sim 2.0 \mathrm{mag}$ brighter than RR Lyrae stars) at periods $\sim 2.0$ days. If compared with other luminous metal-poor variables such as Population II Cepheids (see the recent review by Wallerstein 2002), they appear significantly brighter, at fixed period.

A general consensus exists in the literature (see, e.g., Bono et al. 1997a [B97]; Caputo 1998 and references therein) on the fact that AC variables, like RR Lyrae stars and Population II Cepheids, are metal-poor He-burning stars in the post Zero Age Horizontal Branch (ZAHB) evolutionary phase. As reviewed by Caputo (1998), for low metal abundances $(Z \leq 0.0004)$ and relatively young ages $(\leq 4 \mathrm{Gyr})$ the effective temperature

Send offprint requests to: M. Marconi, e-mail: marcella@na.astro.it of ZAHB models reaches a minimum ( $\left.\log T_{\mathrm{e}} \sim 3.76\right)$ for a mass of about 1.0-1.2 $M_{\odot}$, while if the mass increases above this value, both the luminosity and the effective temperature start increasing, forming the so called "ZAHB turnover". For larger metallicities, the more massive ZAHB structures have brighter luminosities but effective temperatures rather close to the minimum effective temperature. Within such an evolutionary scenario, $\mathrm{AC}$ variables appear to belong to the post-turnover portion of the ZAHB which crosses the instability strip at luminosity higher than RR Lyrae pulsators. Their origin is still debated and the most widely accepted interpretations are: (1) they are young $(\leq 5 \mathrm{Gyr})$ single stars due to recent star formation; (2) they formed from mass transfer in binary systems as old as the other stars in the same stellar system.

The general properties of observed ACs have been analyzed by Nemec et al. (1994) and by Pritzl et al. (2002) who provided empirical Period-Luminosity (PL) relations in the $B$ and $V$ bands for both fundamental (F) and first overtone (FO) pulsators. However, as shown by the quoted authors, the mode assignment for these variables is not an easy task and the behavior in the PL, Period-Amplitude (PA) and Period-Color (PC) planes does not always allow to discriminate between $\mathrm{F}$ and FO pulsation modes.

Based on previous nonlinear convective pulsating models and evolutionary tracks, B97 constrained the blue absolute magnitude and the period of metal-poor central He-burning 
pulsators more massive than RR Lyrae stars, finding a minimum mass for the occurrence of this kind of variables around $\sim 1.3 M_{\odot}$ with $Z=0.0001$ and $\sim 1.8 M_{\odot}$ with $Z=0.0004$. The theoretical limits of the distribution of these high mass pulsators in the $\Delta M_{B}$ vs. $P$ plane, where $\Delta M_{B}$ is the calculated $M_{B}$ scaled to the ZAHB magnitude at the mean effective temperature of the RR Lyrae strip, conformed well to the observed data of most ACs and suggested a minimum mass of about $1.5 M_{\odot}$. According to the position in the PL and PA planes, B97 confirmed that observed ACs are a mix of F and FO pulsators and derived two distinct non parallel $B$ band PL relations for the two pulsation modes. These relations were significantly different from the empirical parallel ones derived by NNL, whereas they better conform with the analysis by Nemec et al. (1988) and Pritzl et al. (2002).

However, B97 pulsation models did not include the most recent input physics and covered a restricted range of stellar parameters. For this reason, we computed new models including updated opacity evaluations and spanning a wider range of stellar parameters. In this paper we present the results of these computations and some preliminary tests of the predictive capability of the updated pulsational scenario.

The organization of the paper is the following: in Sect. 2 we present the models, the results concerning the topology of the instability strip and the light curves. The transformation from theoretical to observational quantities is presented in Sect. 3, together with a short discussion of the different types of mean magnitudes and colors. In the same section, we give the relations connecting pulsational parameters (periods, amplitudes) to absolute magnitudes and colors. The comparison with observed ACs in dwarf spheroidal galaxies is briefly discussed in Sect. 4 and some concluding remarks close the paper.

\section{The pulsation models}

\subsection{Periods and instability strip}

The basic physics underlying radial pulsation, that is the conversion of thermal energy into kinetic energy, is quite well known (e.g., see the nice review by Madore \& Freedman 1991) and, as early shown by Sandage (1958), the natural consequence of the Ritter's period-mean density relation and of the Stefan's law connecting luminosity, radius and effective temperature is that the pulsation period is a function of the luminosity, mass and effective temperature of the pulsator. Present models are computed using a nonlinear, nonlocal and timedependent convective hydrodynamical code which has been already discussed in a series of previous papers (see, e.g., Bono \& Stellingwerf 1994; Bono et al. 1997a,b) and is not repeated here. It seems of importance only to note that, with respect to linear envelope models, the nonlinear approach provides many more predictions beside the pulsation period, such as the luminosity variation along the pulsation cycle, namely the bolometric light curve, and its morphology. Moreover, the inclusion of convection, which tends to quench pulsation, is fundamental to gain reliable information on both the blue and red edges of the pulsation region. Even though we are aware that further computations are needed to refine our knowledge of the pulsation
Table 1. Input parameters for the grid of AC pulsation models with $Y=0.24$ and $Z=0.0001$ and 0.0004 .

\begin{tabular}{lc}
\hline \hline$M / M_{\odot}$ & $\log L / L_{\odot}$ \\
\hline 1.3 & $1.82,1.92$ \\
1.6 & $1.88,1.98,2.08$ \\
1.9 & $1.98,2.08,2.18$ \\
2.0 & $2.08,2.18,2.28$ \\
2.2 & $2.08,2.28$ \\
\hline
\end{tabular}

phenomenon, our hydrodynamical code and physical assumptions have already shown a nice agreement with several observed properties of classical Cepheid (see Bono et al. 1999b, 1999a, 2000b, 2002; Caputo et al. 2000, 2001; Fiorentino et al. 2002) and RR Lyrae models (see Bono et al. 1997b, 2000a, 2003; Marconi et al. 2003), and we are confident that they can be extended to other types of pulsating stars in order to construct a homogeneous pulsational scenario. It is also important to consider that the study of complex stellar systems, where a variety of pulsating stars can be observed, requires that the same input physics should be adopted for both pulsation and evolutionary computations. For these reasons, as for RR Lyrae and classical Cepheid models, we adopt the updated compilations of the opacity tables (Iglesias \& Rogers 1996) used in modern star model computations. Finally, we have considered two chemical compositions representative of the metalpoor abundances of most of the observed ACs $(Z=0.0001$ and 0.0004 , with $Y=0.24)$, a mixing-length parameter $l / H_{\mathrm{p}}=1.5$, and the mass and luminosity levels suggested by He-burning evolutionary models (see Castellani \& Degl'Innocenti 1995), as reported in Table 1.

For each mass and luminosity, the computations are performed by decreasing the effective temperature $T_{\mathrm{e}}$ by steps of $100 \mathrm{~K}$, and the period from the bluest to the reddest pulsating model is derived ${ }^{1}$. Note that increasing (decreasing) by $100 \mathrm{~K}$ the effective temperature of the bluest (reddest) pulsators yields non-pulsating structures. In analogy with other well studied pulsators such as RR Lyrae stars (see van Albada \& Baker 1971; Bono et al. 1997b; Marconi et al. 2003) and classical Cepheids (see Bono et al. 2000b), linear regression through the results allows us to derive analytical relations connecting the period of both fundamental (F) and first-overtone (FO) modes to the intrinsic stellar parameters, namely mass, luminosity, and effective temperature. We obtain:

$$
\begin{aligned}
\log P_{\mathrm{F}}= & 10.879( \pm 0.001)+0.818 \log L-0.616 \log M \\
& -3.309 \log T_{\mathrm{e}} \\
\log P_{\mathrm{FO}}= & 10.198( \pm 0.001)+0.775 \log L-0.530 \log M \\
& -3.143 \log T_{\mathrm{e}}
\end{aligned}
$$

where mass $M$ and luminosity $L$ are in solar units. As a whole, the period of FO pulsators can be fundamentalised as $\log P_{\mathrm{F}}=$ $\log P_{\mathrm{FO}}+0.13$, in agreement with the procedure currently adopted for RR Lyrae stars.

For fixed metal abundance, mass and luminosity, FO pulsators are generally bluer than F pulsators, so that the blue

\footnotetext{
1 The entire set of results is available upon request to the authors.
} 


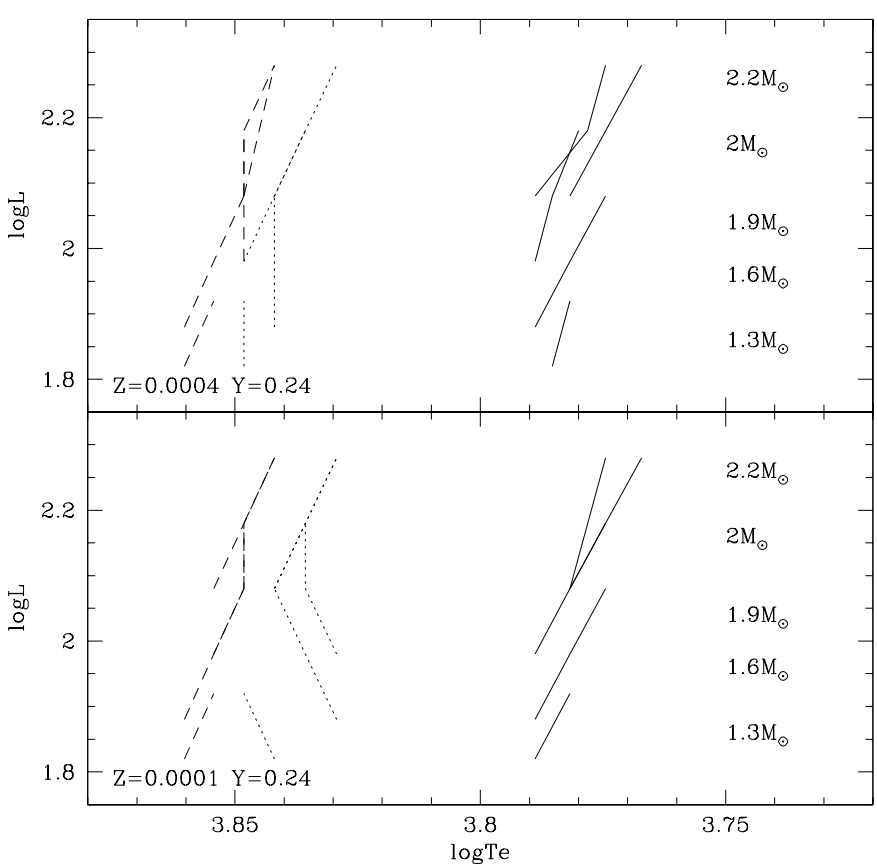

Fig. 1. Dependence of the FOBE (dashed line), FBE (dotted line) and FRE (solid line) on mass and luminosity, for the two labelled metal abundances.

edge of the first-overtone pulsation region (FOBE) and the red edge of the fundamental one (FRE) can be taken as representative of the boundaries of the whole instability strip, at least in the studied range of mass and luminosity. Given the adopted steps of $100 \mathrm{~K}$ in our computations, we adopt as FOBE and FRE the effective temperature of the bluest FO model and of the reddest $\mathrm{F}$ model, increased and decreased by $50 \mathrm{~K}$, respectively, with an intrinsic uncertainty of $\pm 50 \mathrm{~K}$. As for the internal topology of the pulsation region, the computations show that the blue edge for fundamental pulsation (FBE) is bluer than the first-overtone red edge (FORE), suggesting the occurrence of a middle region of the instability strip where both the pulsation modes can be stable, similarly to what found for RR Lyrae stars (see van Albada \& Baker 1973; Marconi et al. 2003 and references therein). Moreover, we find that the effective temperature of reddest FO models, at fixed mass and luminosity, appears to be sensitive to the location of the pulsation node within the convective stellar envelope, deserving a detailed analysis that will be presented in a forthcoming paper.

The lines drawn in Fig. 1 show, left to right, the location in the HR diagram of the bluest FO, the bluest F and the reddest F pulsators, respectively, for the labelled mass and varying the luminosity under the assumption $l / H_{\mathrm{p}}=1.5$. We find that, at constant mass and luminosity, a variation of $Z$ from 0.0001 to 0.0004 has a marginal influence on the instability strip topology, the only evident effect being the narrowing of the region where only FO pulsation is efficient, that is the region between the bluest FO and F models, when passing from $Z=0.0001$ to $Z=0.0004$. As a whole, in spite of the different opacity tables, the predicted topology of the instability strip is similar to the one found by B97 for a stellar mass $1.5 M_{\odot}$ and $Z=0.0001$ (see Fig. 1 in B97).
Inspection of the results plotted in Fig. 1 shows that the entire pulsation region tends to move towards the blue if increasing the mass (at constant luminosity) or decreasing the luminosity (at constant mass). A linear regression to the results yields that, in the range $Z=0.0001$ to 0.0004 , the limits of the AC instability strip can be approximated as

$$
\begin{aligned}
\log T_{\mathrm{e}}(\mathrm{FOBE})= & 3.947( \pm 0.006)-0.052 \log L \\
& +0.042 \log M \\
\log T_{\mathrm{e}}(\mathrm{FRE})= & 3.898( \pm 0.006)-0.065 \log L \\
+ & 0.058 \log M
\end{aligned}
$$

where the intrinsic uncertainty of $\pm 50 \mathrm{~K}$ on the FOBE and FRE temperatures is included. Concerning the fundamental blue edge (FBE), our models suggest $\log T_{\mathrm{e}}(\mathrm{FBE})-$ $\log T_{\mathrm{e}}(\mathrm{FRE}) \sim 0.055 \pm 0.005$, for fixed mass and luminosity. Note that, as already discussed for RR Lyrae stars (see Caputo et al. 2000; Marconi et al. 2003), predictions concerning the FOBE, that is the boundary between non pulsating and pulsating stars at the hotter effective temperatures, are particularly solid. In fact, the uncertainties connected to the treatment of convection are expected to be smaller in the blue region (high effective temperatures) of the instability strip and specific tests show that a variation of the mixing length parameter from $l / H_{\mathrm{p}}$ from 1.5 (the adopted value in this investigation) to 2.0 implies that the FOBE becomes redder by not more than $100 \mathrm{~K}$ whereas the FRE becomes bluer by at least $200 \mathrm{~K}$.

\subsection{Light curves and pulsation amplitudes}

For each investigated model, the variation of relevant parameters (luminosity, radius, radial velocity, effective temperature and gravity) along a pulsation cycle was provided. A sub-sample of bolometric light curves for $Z=0.0001$ (with $M / M_{\odot}=2.0$ and $\log L / L_{\odot}=2.08$ ) is shown in Figs. 2a,b for fundamental and first overtone pulsators, respectively. The behavior of the curves shows that, at variance with the case of RR Lyrae stars, no clear information on the pulsation mode can be drawn from the light curve morphology. Indeed, even if the raising branch is steeper in the case of the fundamental mode, the light curves for both modes present a variety of morphological features, deviating from a sinusoidal shape but for the few hottest first overtone pulsators.

Figure 3 shows bolometric amplitudes versus periods for selected models with $Z=0.0001$. In particular, the effects of varying the luminosity level at fixed stellar mass (2.2 $M_{\odot}$, top panel) and of varying the mass at fixed luminosity $\left(\log L / L_{\odot}=2.08\right.$, bottom panel) are shown. As a whole, fundamental pulsators follow the well known behavior of fundamental RR Lyrae stars, with the amplitude increasing as the period decreases, for fixed mass and luminosity. Also the dependence on mass and luminosity is the same as found for RR Lyrae stars (see Bono et al. 1997b; Marconi et al. 2003): at fixed period, the amplitude increases with increasing the luminosity or decreasing the mass. Moreover, the rather linear dependence of the amplitude with period will allow us (see Sect. 3) to derive fundamental Period-Amplitude relations in analogy with RR Lyrae stars. 

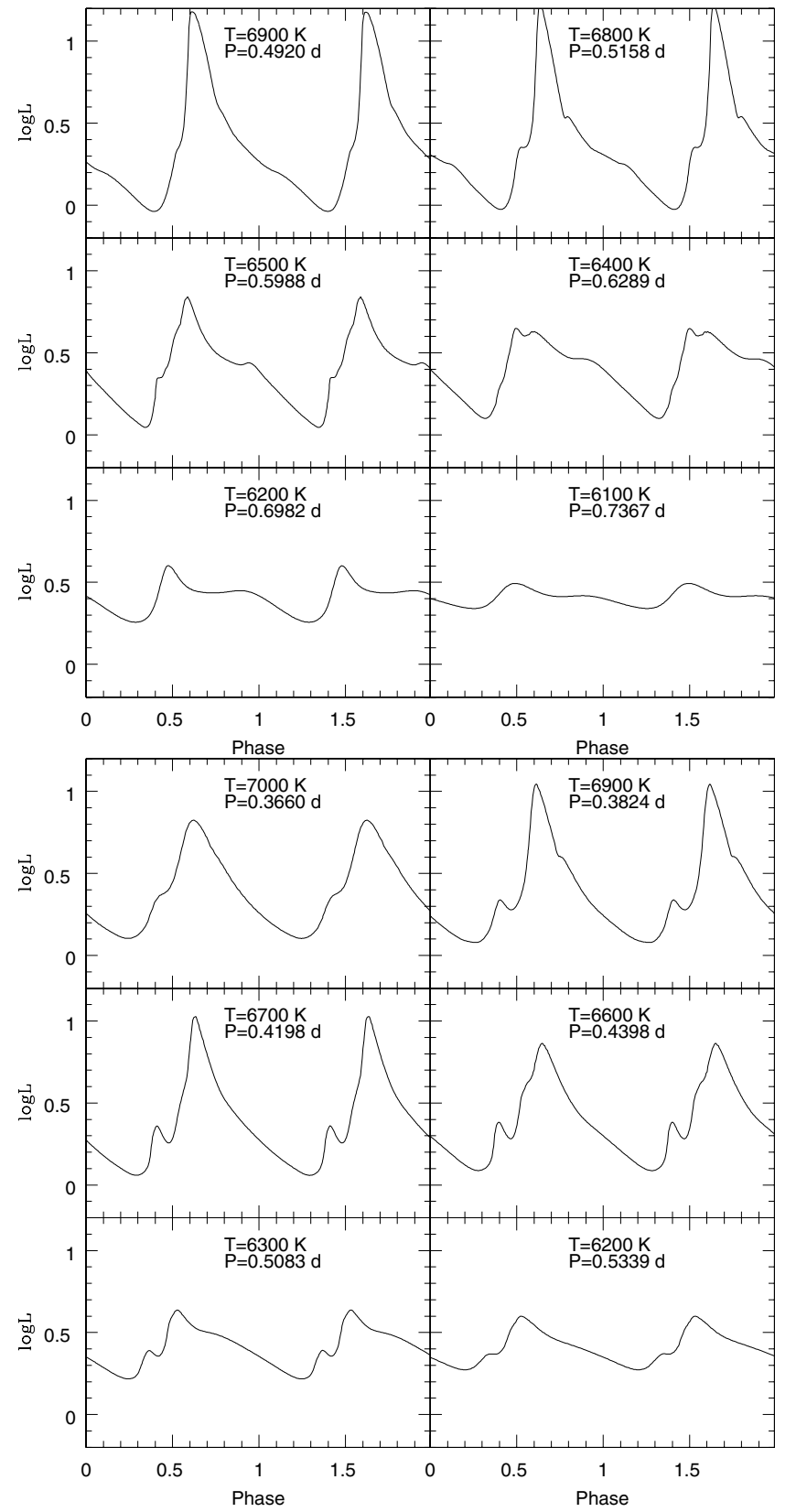

Fig. 2. Theoretical bolometric light curves for a subsample $\left(M=2 M_{\odot}\right.$ $\log L / L_{\odot}=2.08$ ) of fundamental a) and first overtone b) models with $Z=0.0001$.

As for first overtone pulsators, let us recall that, for any fixed mass and luminosity, a model with an effective temperature $100 \mathrm{~K}$ higher than the hottest pulsating model is not pulsating. This means that within $\delta \log P \sim 0.02-0.03$ to the left of the hottest (shortest period) FO model plotted in Fig. 3 the amplitude should reach vanishing values. On this basis, the amplitudes plotted in Fig. 3 appear to show the characteristic bell shape typical of first overtone RR Lyrae stars.

However, in spite of these similarities, two main differences appear to exist between the behaviors of ACs and RR Lyrae stars in the Bailey diagram:

1. in the case of ACs the maximum amplitudes reached by first overtone pulsators may be comparable with those of

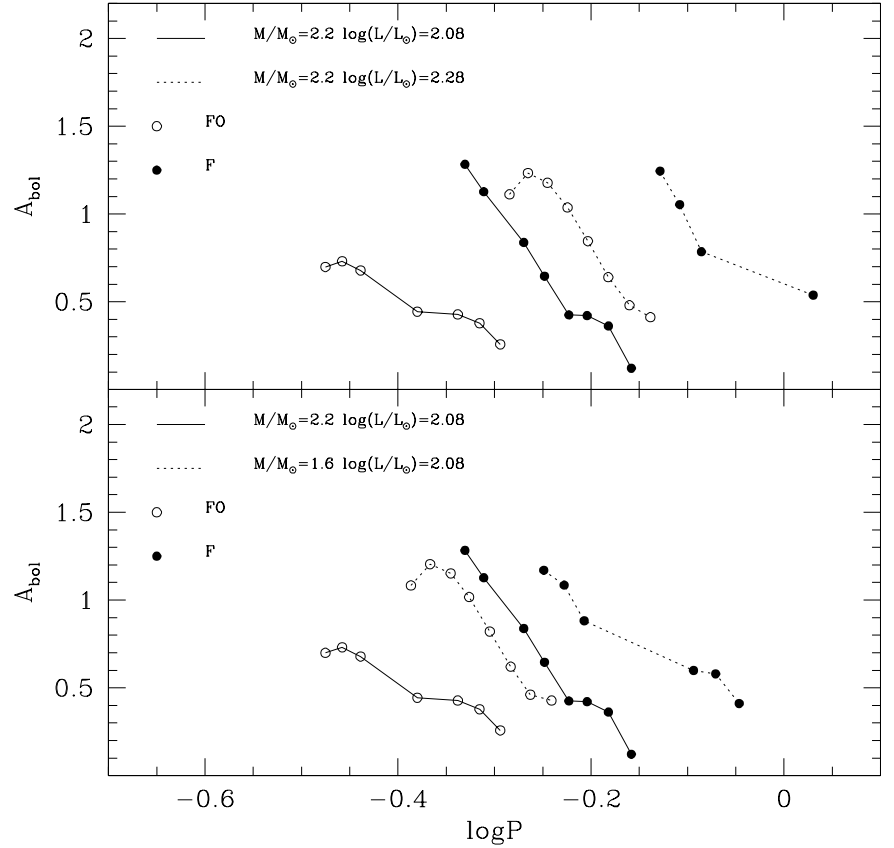

Fig. 3. Bolometric amplitudes versus periods for selected models with $Z=0.0001$ at varying the luminosity level at fixed stellar mass $\left(2.2 M_{\odot}\right.$, top panel) and the mass at fixed luminosity $\left(\log L / L_{\odot}=2.08\right.$, bottom panel).

fundamental ones, whereas fundamental RR Lyrae pulsators always attain significantly higher amplitudes;

2. the maximum of the bell defined by first overtone pulsators decreases when the stellar mass increases or the luminosity level decreases, following an opposite trend with respect to first overtone RR Lyrae stars (see Bono et al. 1997b, for details).

Considering that observed ACs, even in the same stellar system, may have different mass and luminosity, with the more massive ones at brighter luminosities, one has that the discrimination of the AC pulsation mode from the analysis of the period-amplitude diagram might be not an easy task. As a fact, inspection of Fig. 3 reveals that the periodamplitude distribution of the more massive $\left(2.2 M_{\odot}\right)$ and brighter $\left(\log L / L_{\odot}=2.28\right) \mathrm{FO}$ pulsators (dotted line connecting open circles in the top panel), is quite close to that of less massive $\left(1.6 M_{\odot}\right)$ and fainter $\left(\log L / L_{\odot}=2.08\right) \mathrm{F}$ pulsators (solid line connecting dots in the bottom panel).

\section{From theoretical to observational quantities}

In order to compare theoretical results with observations, the bolometric light curves have to be transformed into the photometric bands $U B V R I J K$. As in previous studies performed by our group, we use bolometric corrections and temperaturecolor relations provided by Castelli et al. (1997a,b).

Before proceeding, it is worth noticing that for observed pulsating stars we can only measure magnitudes and colors that are time-averaged quantities over a pulsation period, rather than the static values the stars would have were they not pulsating. For this reason, in consideration that observed variables 


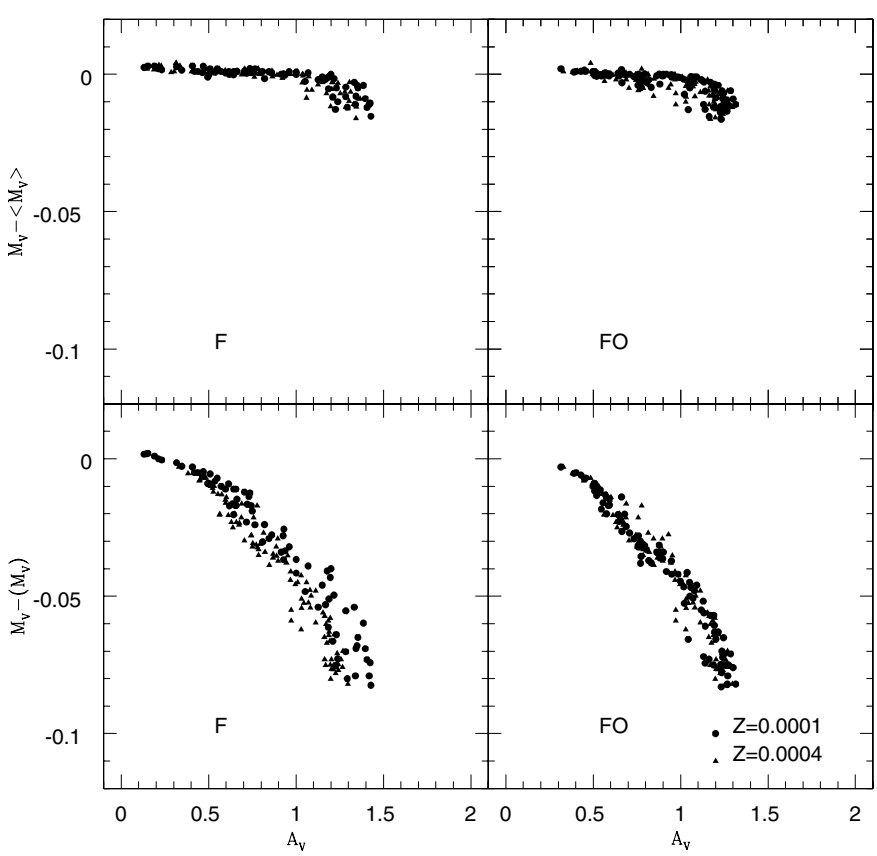

Fig. 4. Difference between static $M_{V}$ and either intensity weighted (top panels) or magnitude averaged (bottom panels) mean magnitudes as a function of the pulsation amplitude for both fundamental (F) and first overtone (FO) models.

are fitted with evolutionary tracks, it is important to estimate the mean values from pulsation models and compare them with the corresponding static quantities. A similar study has already been performed for RR Lyrae stars (Bono et al. 1995; Marconi et al. 2003) and classical Cepheids (Caputo et al. 1999) and we are now in the position to extend the analysis to ACs.

With $<M_{i}>$ and $\left(M_{i}\right)$ indicating intensity-weighted and magnitude-weighted mean magnitudes, respectively, we show in Fig. 4 the difference between static $M_{V}$ and mean magnitudes as a function of the pulsation visual amplitude $A_{\mathrm{V}}$. One has that intensity-weighted means are better representative of the static values than magnitude-averaged quantities, in both cases the discrepancy increasing toward higher amplitudes and less symmetric light curves. As far as the $B V$ color is concerned, Fig. 5 shows that increasing the visual amplitude the magnitude-averaged mean colors $(B-V)$ tend to be redder than the corresponding static values, whereas the opposite behavior holds for the intensity-weighted mean colors $\langle B\rangle-\langle V\rangle$.

In general, the behaviors found for present models are in agreement with the results already found for RR Lyrae stars and classical Cepheids. In analogy with the procedure followed by Marconi et al. (2003) for RR Lyrae stars, we derive the predicted relation to estimate static $V_{\mathrm{s}}$ magnitudes from observed magnitude and intensity averaged values, as given by

$V_{\mathrm{s}}=1.20<V>-0.20(V)$

with an uncertainty of \pm 0.01 mag. This relation appears in substantial agreement with the results recently presented for RR Lyrae models with $Z=0.001$ (see Marconi et al. 2003).

However, bearing in mind that the pulsation amplitude of models close to the boundaries (FOBE and FRE) of the instability strip approaches the zero value, the location of FOBE

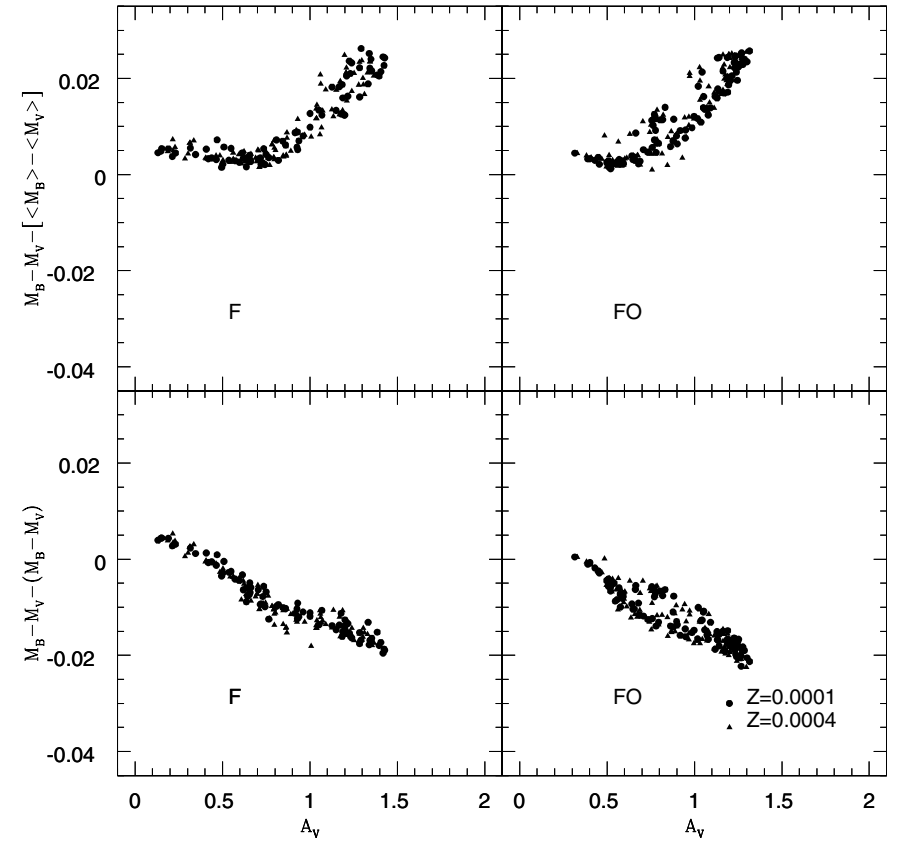

Fig. 5. The same but for the $B-V$ color.

and FRE in the $M_{V}-\log P$ plane turns out to be quite independent of whether static or mean magnitudes are considered. As shown in Fig. 6, a linear regression to the models with $l / H_{\mathrm{p}}=1.5$ yields the analytical relations

$$
\begin{aligned}
& M_{V}(\mathrm{FOBE})=-1.12-2.68 \log P_{\mathrm{FO}}-1.80 \log M \\
& M_{V}(\mathrm{FRE})=-0.03-2.32 \log P_{\mathrm{F}}-1.92 \log M
\end{aligned}
$$

with $\sigma_{V}= \pm 0.06 \mathrm{mag}$, including the intrinsic $\pm 50 \mathrm{~K}$ uncertainty on FOBE and FRE effective temperatures. Adopting $l / H_{\mathrm{p}}=2.0$, the zero-point in Eqs. (5) and (6) changes respectively by $\sim+0.1 \mathrm{mag}$ and $\sim-0.18 \mathrm{mag}$. As for the blue limit of fundamental pulsation (FBE), where the amplitude reaches quite large values, we consider intensity-weighted magnitudes, deriving that the predicted location of the FBE can be approximated as

$<M_{V}(\mathrm{FBE})>=-0.72-2.61 \log P_{\mathrm{F}}-1.71 \log M$

with $\sigma_{V}= \pm 0.07 \mathrm{mag}$.

\subsection{Period-Magnitude-Color, Color-Color relations and Wesenheit functions}

The natural outcome of the period relation (Eqs. (1a) and (1b)) in the observational plane is the Period-Magnitude-Color relation (hereafter named PMC) in which the period is correlated with the pulsator absolute magnitude and color, for any given mass. The coefficients of the intensity-weighted PMC relations in the various bands are reported in Table 2, while Fig. 7 shows the quite small scatter of the models around the predicted relations.

According to these relations, one could estimate the mass range spanned by a sample of ACs at the same distance and with the same reddening with a formal uncertainty of $\sim 2 \%$, 


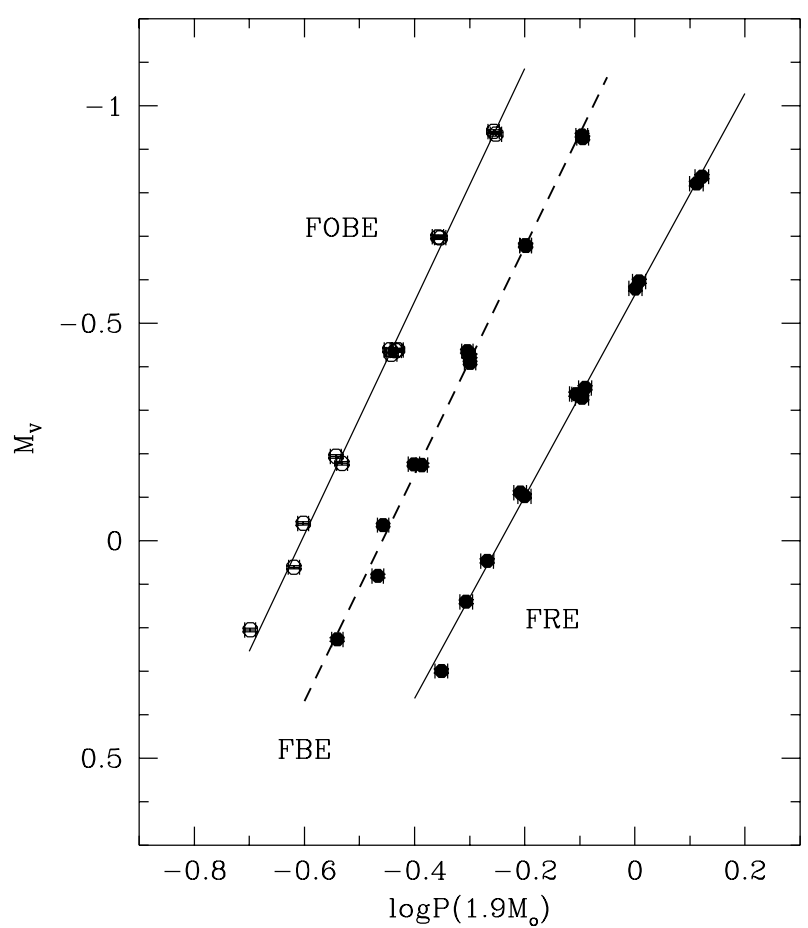

Fig. 6. Limits of the pulsation region (FOBE and FRE) and blue limit for fundamental pulsation (FBE) in the $M_{V}-\log P$ plane. The periods are normalized to the same mass $\left(1.9 M_{\odot}\right)$, according to the predicted dependence (see text).

Table 2. Coefficients of the mass-dependent Period-Magnitude-Color relations.

\begin{tabular}{|c|c|c|c|c|c|}
\hline Mode & $\alpha$ & $\beta$ & $\gamma$ & $\delta$ & $\sigma_{V}$ \\
\hline \multicolumn{6}{|c|}{$<M_{V}>=\alpha+\beta \log P+\gamma[<B>-<V>]+\delta \log M$} \\
\hline $\mathrm{F}$ & -1.56 & -2.85 & 3.51 & -1.88 & 0.01 \\
\hline FO & -1.92 & -2.90 & 3.43 & -1.82 & 0.02 \\
\hline \multicolumn{6}{|c|}{$<M_{V}>=\alpha+\beta \log P+\gamma[<V>-<R>]+\delta \log M$} \\
\hline $\mathrm{F}$ & -1.69 & -2.90 & 5.39 & -1.89 & 0.01 \\
\hline FO & -2.09 & -3.05 & 5.28 & -1.75 & 0.01 \\
\hline \multicolumn{6}{|c|}{$<M_{V}>=\alpha+\beta \log P+\gamma[<V>-<I>]+\delta \log M$} \\
\hline $\mathrm{F}$ & -1.83 & -2.93 & 2.73 & -1.89 & 0.01 \\
\hline FO & -2.24 & -3.09 & 2.67 & -1.73 & 0.01 \\
\hline \multicolumn{6}{|c|}{$<M_{V}>=\alpha+\beta \log P+\gamma[<V>-<J>]+\delta \log M$} \\
\hline $\mathrm{F}$ & -1.95 & -3.01 & 1.78 & -1.87 & 0.01 \\
\hline FO & -2.38 & -3.17 & 1.75 & -1.70 & 0.01 \\
\hline \multicolumn{6}{|c|}{$<M_{V}>=\alpha+\beta \log P+\gamma[<V>-<K>]+\delta \log M$} \\
\hline $\mathrm{F}$ & -1.99 & -3.05 & 1.31 & -1.86 & 0.01 \\
\hline FO & -2.40 & -3.22 & 1.29 & -1.68 & 0.01 \\
\hline \multicolumn{6}{|c|}{$<M_{V}>=\alpha+\beta \log P+\gamma[<J>-<K>]+\delta \log M$} \\
\hline $\mathrm{F}$ & -1.97 & -3.07 & 2.17 & -1.85 & 0.01 \\
\hline FO & -2.41 & -3.24 & 2.11 & -1.66 & 0.01 \\
\hline
\end{tabular}

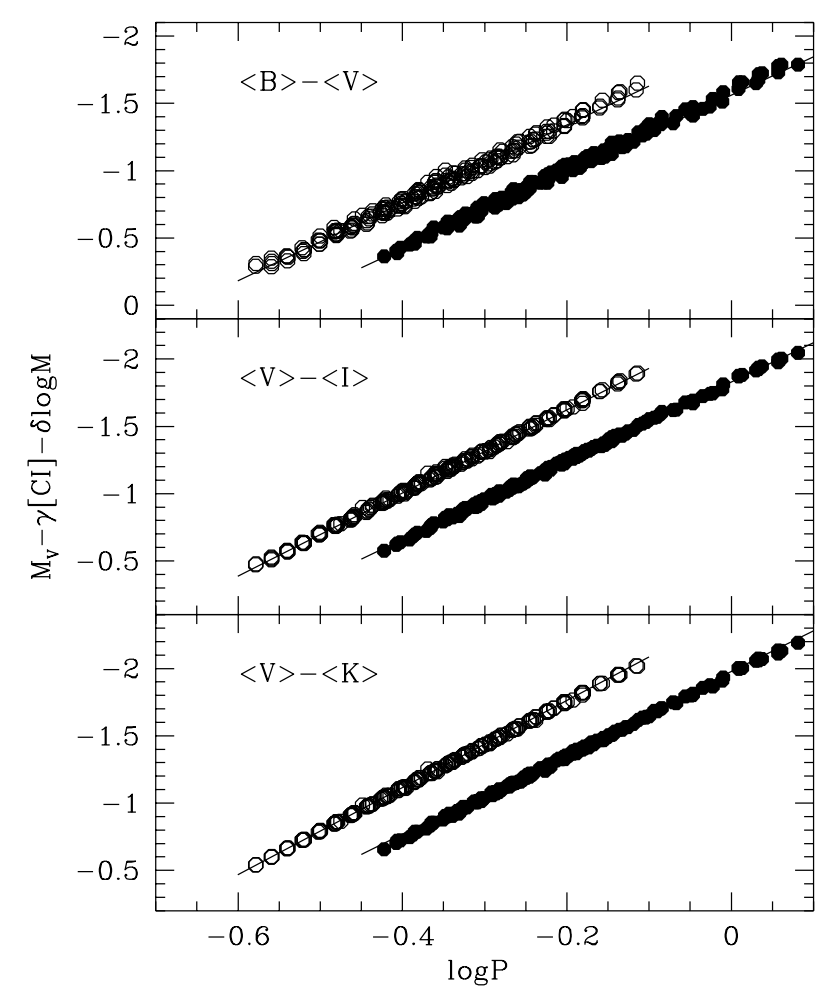

Fig. 7. PMC relations in the labelled photometric bands for both fundamental (filled circles) and first overtone (open circles) models. The solid line represents the linear regression through the data (see Table 2).

provided that periods and colors are well measured. If the distance and reddening are independently known, then the mass absolute values can be determined too.

We have also investigated the relations among intensityaveraged colors, from optical to near infrared bands. As given in Table 3, these relations have an intrinsic dispersion of the order of few thousands of magnitude, but unfortunately they appear to be of no use for what concerns the reddening determination. As a fact, apart from the $\langle B\rangle-\langle V\rangle$ versus $\langle V\rangle-\langle R\rangle$ case, the slope $b$ of the predicted relations is very close to the reddening vector $\boldsymbol{r}$, as estimated using a typical extinction law (see e.g. Cardelli et al. 1989).

In order to complete the pulsational framework, we have finally considered the reddening free Wasenheit functions (Madore 1982) and their dependence on periods. As already discussed in several papers on classical Cepheids (see, e.g., Madore \& Freedman 1991; Tanvir 1999; Caputo et al. 2000), the intrinsic variation due to the finite width of the instability strip is almost similar to the effects of interstellar extinction, and the color term in the Wasenheit functions $[W(B, V)=$ $M_{V}-3.1(B-V), W(V, I)=M_{V}-2.54(V-I)$, etc.], thought to cancel the reddening effect, helps to reduce the dispersion of magnitudes at a given period. On this basis, the PeriodWesenheit relations (hereafter referred to as PW) is used to get reliable estimates of the intrinsic distance modulus.

This effect is shown in Fig. 8, while the coefficients of the predicted intensity weighted $\mathrm{PW}$ relations, as derived by fundamental models and first-overtone models with fundamentalised periods, are reported in Table 4 for selected photometric bands. 


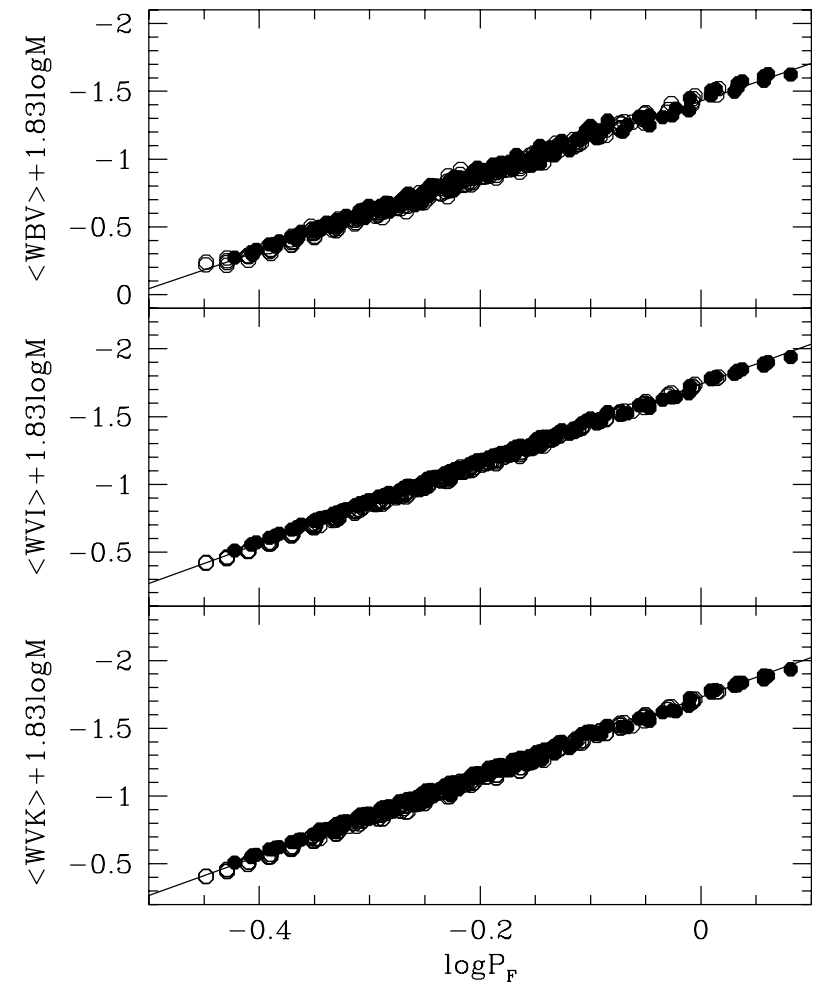

Fig. 8. PW relations in the labelled photometric bands for fundamental (filled circles) and first overtone (open circles) models with fundamentalised periods. The solid line represents the linear regression through the data (see Table 4).

Table 3. Coefficients of intensity averaged Color-Color relations for fundamental models at $Z=0.0001$. The last column gives the reddening vector, as estimated using a typical extinction law (see text).

\begin{tabular}{|c|c|c|c|c|}
\hline Mode & $a$ & $b$ & $\sigma$ & $r$ \\
\hline \multicolumn{5}{|c|}{$<B>-<V>=a+b[<V>-<R>]$} \\
\hline $\mathrm{F}$ & -0.027 & 1.509 & 0.002 & 2.03 \\
\hline FO & -0.016 & 1.461 & 0.004 & 2.03 \\
\hline \multicolumn{5}{|c|}{$<B>-<V>=a+b[<V>-<I>]$} \\
\hline $\mathrm{F}$ & -0.060 & 0.755 & 0.002 & 0.82 \\
\hline FO & -0.046 & 0.726 & 0.004 & 0.82 \\
\hline \multicolumn{5}{|c|}{$<B>-<V>=a+b[<V>-<J>]$} \\
\hline $\mathrm{F}$ & -0.077 & 0.478 & 0.004 & 0.45 \\
\hline FO & -0.062 & 0.461 & 0.007 & 0.45 \\
\hline \multicolumn{5}{|c|}{$<B>-<V>=a+b[<V>-<K>]$} \\
\hline $\mathrm{F}$ & -0.070 & 0.347 & 0.005 & 0.36 \\
\hline FO & -0.056 & 0.335 & 0.008 & 0.36 \\
\hline
\end{tabular}

Note that the use of these reddening-free relations to get the mass range of a sample of ACs at the same distance yields a formal uncertainty of $\sim 5 \%$, provided that periods and colors are well measured. On the other hand, if the mass of the variable is independently known, these relations can provide its intrinsic distance modulus.
Table 4. Coefficients of selected mass-dependent fundamental PW relations $\left\langle W>=\alpha+\beta \log P_{\mathrm{F}}+\gamma \log M\right.$.

\begin{tabular}{|c|c|c|c|}
\hline$\alpha$ & $\beta$ & $\gamma$ & $\sigma_{V}$ \\
\hline \multicolumn{4}{|c|}{$<W(B, V)>=<M_{V}>-3.10[<B>-<V>]$} \\
\hline-1.43 & -2.77 & -1.83 & 0.04 \\
\hline \multicolumn{4}{|c|}{$<W(V, I)>=<M_{V}>-2.54[<V>-<I>]$} \\
\hline-1.74 & -2.94 & -1.83 & 0.04 \\
\hline \multicolumn{4}{|c|}{$<W(V, K)>=<M_{V}>-1.13[<V>-<K>]$} \\
\hline-1.73 & -2.93 & -1.83 & 0.04 \\
\hline
\end{tabular}

\subsection{Period-Magnitude-Amplitude relation}

Once the bolometric light curves are transformed into the observational plane, pulsation amplitudes in the various photometric bands are derived. As already noticed in Sect. 2, the behavior of fundamental pulsators in the Period-Amplitude diagram is rather linear, but with a non negligible dependence on both the pulsator mass and luminosity. This occurrence suggests the possibility to derive a mass-dependent relation between period, absolute magnitude and amplitude (PMA). A linear regression through the fundamental models with $Z=0.0001$ and 0.0004 gives the relation (see also Fig. 9)

$\log P_{\mathrm{F}} *=\log P_{\mathrm{F}}+0.41<M_{V}>+0.77 \log M=$

$$
0.01( \pm 0.05)-0.188 A_{\mathrm{V}}
$$

that again should allow to estimate the mass range spanned by fundamental ACs at the same distance and with well measured periods and amplitudes, but with a formal uncertainty of $\sim 15 \%$ which is larger than that related with the PMC and PW relations. However, it is of interest to note that, since the PMA relation only holds for fundamental pulsators, a comparison with the results inferred from the PMC or PW relations should provide a way to discriminate the pulsation mode of observed ACs (see below).

\section{Comparison with observations}

\subsection{Anomalous Cepheids in dwarf spheroidal galaxies}

As clearly shown by the evolutionary models (see Castellani \& Degl'Innocenti 1995, B97, and the recent Pisa (www. mporzio.astro.it/marco/GIPSY/homegipsy.html) and Padova (http://pleiadi.pd.astro.it) libraries), metalpoor central He-burning structures more massive than $\sim 1.3 M_{\odot}$ evolve with a characteristic "blue loop" whose luminosity and effective temperature increases when increasing the mass. Since the effective temperature at the edges of the instability strip is a function of mass and luminosity, it follows that one can easily estimate whether a star with a given mass is expected to cross the pulsation region becoming a variable star. Moreover, since at fixed metal content the He-burning pulsators of a given mass would have a quite defined range of intrinsic luminosity, the mass-term in the above predicted relations could be removed to provide "evolutionary" relations. 


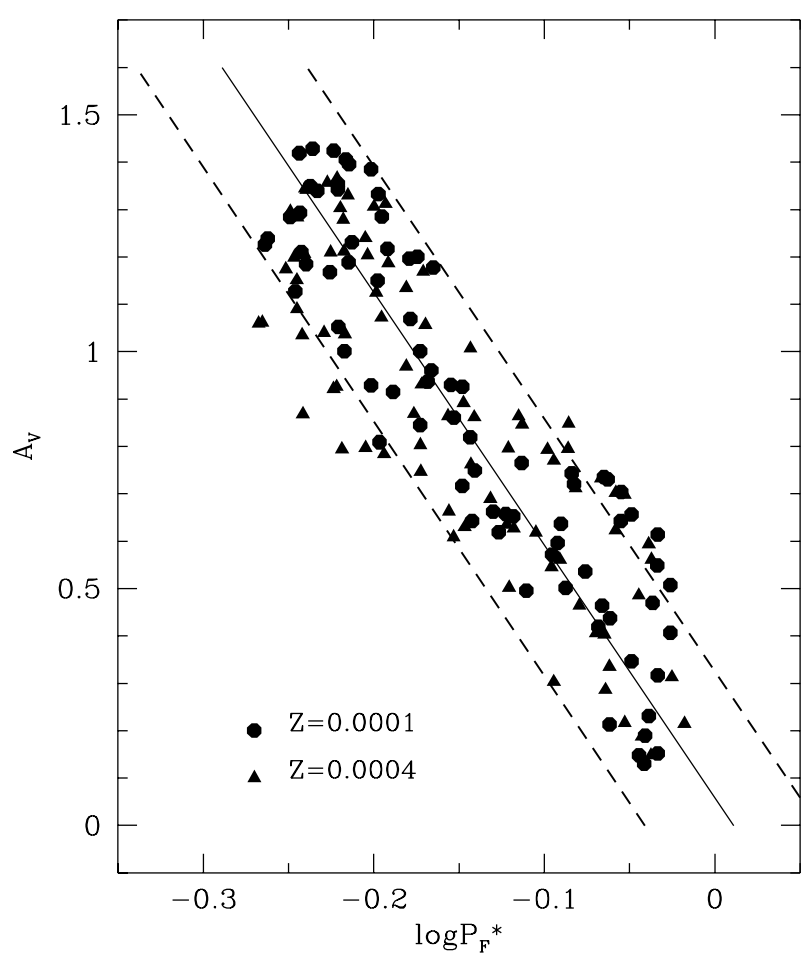

Fig. 9. PMA relations for fundamental models. The solid line represents the linear regression through the data (see Eq. (8)).

However, from the point of view of stellar evolution one should consider that the mass/luminosity relation of central Heburning structures is dependent on the metal content, as well as that, even in presence of stellar models computed with the most updated input physics, the He-burning luminosity of a given mass depends on the adopted efficiency of core overshooting. As shown in Fig. 10 for two selected mass values, canonical He-burning models computed with an inefficient overshooting (Pisa library) are brighter by $\sim 0.25$ mag with respect to overshooting models (Girardi et al. 2000). However, if for the given mass the predicted FRE is taken into consideration, then the inclusion of overshooting seems to leave almost unvaried the minimum mass for the occurrence of $\mathrm{AC}$ variables. On this basis, in this paper we wish to present only the results of pulsation models, leaving mass and luminosity as free parameters and deserving to a forthcoming paper the connection to stellar evolution theory and the construction of synthetic relations based also on the evolutionary times.

In this section, we are going to apply the theoretical relations presented in the previous section to observed AC samples in dwarf spheroidal galaxies with the purpose to estimate their mass. We searched in the literature for bona fide AC pulsators with well measured periods, magnitudes and amplitudes and we found the samples reported in Table 5 where the columns give the host galaxy, the number of measured ACs, the available observed quantities, the intrinsic distance modulus $\mu_{0}$, the $E(B-V)$ reddening (both $\mu_{0}$ and $E(B-V)$ as listed by Pritzl et al. 2002) and the corresponding references.

A first comparison between theoretical results and observations is presented in Fig. 11 where observed ACs are plotted in the $M_{V}-\log P$ diagram together with the predicted boundaries

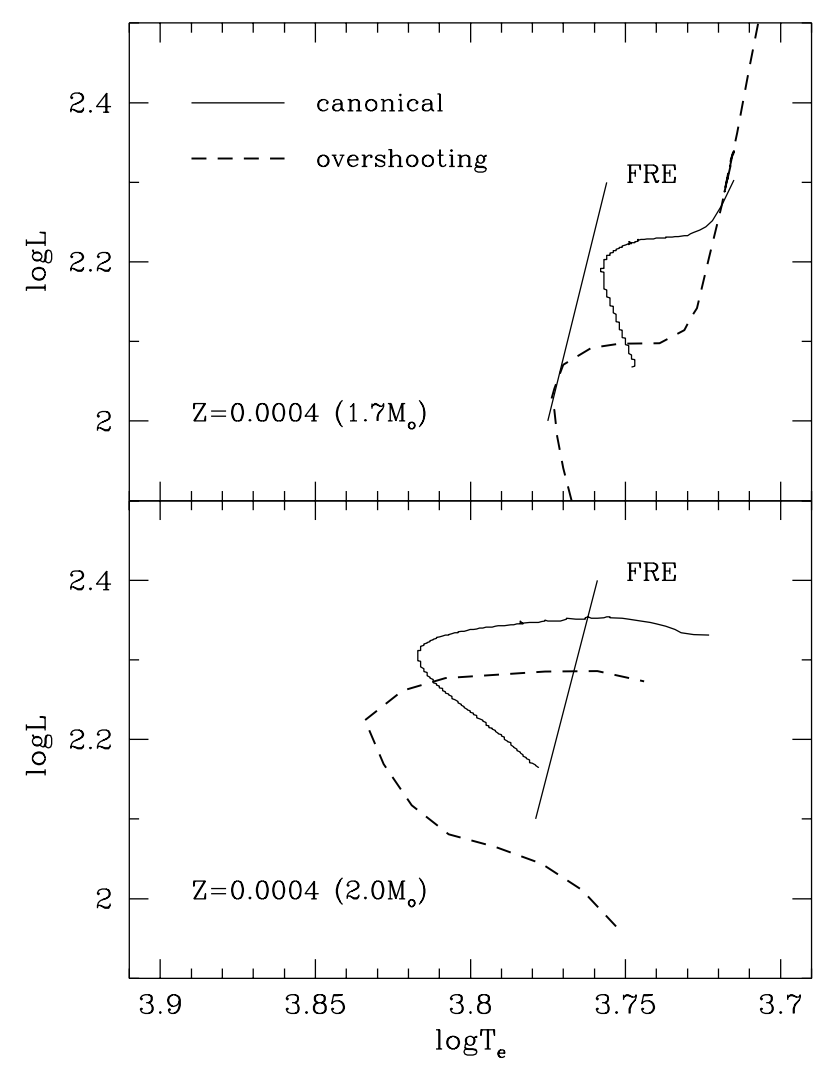

Fig. 10. Central He-burning evolutionary tracks for the labelled masses and metal content as computed without (solid line) or with overshooting (dashed line). The predicted FRE is also shown.

(FOBE and FRE) of the instability strip at $1.3 M_{\odot}$ (dashed line) and $2.2 M_{\odot}$ (solid line). We notice that the general behavior of observed pulsators is in substantial agreement with theory. Specifically, the comparison between the observed distribution and the predicted edges confirms that the brightest variables should have larger masses than the faintest ones.

For almost all the dwarf galaxies reported in Table 5, except Carina and Fornax, a mode discrimination has been already attempted (see Pritzl et al. 2002), as listed in the first column of Table 6 . Based on this classification and using the intrinsic distance moduli and reddening values listed in Table 5, we proceed in estimating the mass of individual variables from the predicted PMC relations given in Table 2. The results are given in Cols. (7) and (8) in Table 6. Furthermore, for F pulsators only, the mass is estimated also from the predicted PMA relation (see Eq. (8)), yielding the values listed in Col. (9) in Table 6. It seems worth noticing that the stellar masses inferred in this paper appear in substantial agreement with the mass range of ACs, as predicted by evolutionary models of not-tooold metal-poor HB structures (see Castellani \& Degl'Innocenti 1995; B97, and the quoted Pisa and Padova libraries).

The mass values listed in Table 6 for the fundamental pulsators are plotted in the upper panel in Fig. 12, together with the formal errors as given by the intrinsic dispersion of the predicted PMC and PMA relations. The same intrinsic dispersions yield that the two mass values should agree at a level of $\delta \log M \sim \pm 0.07$ (solid lines). Allowing for further uncertainties as due to photometric errors and light curve fitting, the 
Table 5. Selected parameters of anomalous Cepheids in dwarf spheroidal galaxies.

\begin{tabular}{lccccc}
\hline \hline Host galaxy & AC number & Observed quantities & $\mu_{0}$ & $E(B-V)$ & Source \\
\hline And VI & 6 & $V, B, A_{\mathrm{V}}$ & $24.45 \mathrm{mag}$ & $0.06 \mathrm{mag}$ & Pritzl et al. (2002) \\
Leo II & 4 & $V, A_{\mathrm{V}}$ & $21.59 \mathrm{mag}$ & $0.02 \mathrm{mag}$ & Pritzl et al. (2002) \\
Draco & 8 & $V, B, A_{\mathrm{V}}$ & $19.49 \mathrm{mag}$ & $0.03 \mathrm{mag}$ & Pritzl et al. (2002) \\
Carina & 8 & $V, B, A_{\mathrm{V}}$ & $20.14 \mathrm{mag}$ & $0.04 \mathrm{mag}$ & Dall'Ora et al. (2003) \\
Sculptor & 3 & $V, A_{\mathrm{V}}$ & $19.56 \mathrm{mag}$ & $0.02 \mathrm{mag}$ & Kaluzny et al. (1995) \\
Sextans & 6 & $V, B, A_{\mathrm{V}}$ & $19.74 \mathrm{mag}$ & $0.03 \mathrm{mag}$ & Mateo et al. (1995) \\
Fornax & 17 & $V, I$ & $20.70 \mathrm{mag}$ & $0.03 \mathrm{mag}$ & Bersier \& Wood (2002) \\
\hline
\end{tabular}

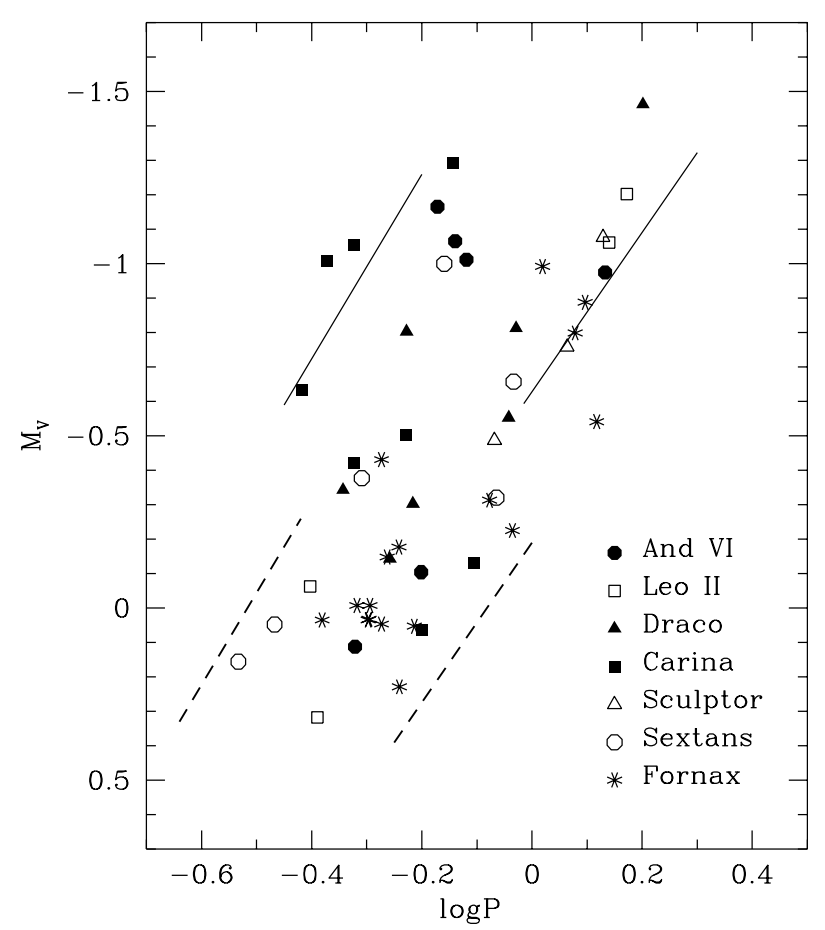

Fig. 11. Comparison between the location of observed ACs in the $M_{V}-\log P$ diagram and the predicted boundaries of the instability strip at $1.3 M_{\odot}$ (dashed line) and $2.2 M_{\odot}$ (solid line).

results plotted in the upper panel in Fig. 12 confirm that for all the fundamental candidates, except V157 in Draco (FO pulsator?), the mass values predicted by the two relations agree with each other within the errors, even though the mass suggested by the PMC relation appears systematically smaller ( $\Delta \log M \sim-0.09$ ) than the PMA results. This effect could be connected to the theoretical uncertainty on the $l / H_{\mathrm{p}}$ parameter which affects pulsation amplitudes and in turn the PMA relation. This also means that one could in principle calibrate this free parameter by requiring the agreement between the PMA results and those inferred by the PMC, which is independent of the adopted $l / H_{\mathrm{p}}$ value. Finally, it is of interest to notice that the difference between the two mass values is independent of errors on the apparent distance modulus, whereas it depends on the adopted reddening. Moreover, the mass absolute values listed in Table 6 should vary by about $\pm 12 \%$ with $\delta \mu= \pm 0.1$ mag.

Let us now assume that also the FO variables are actually fundamental pulsators. Using again the fundamental PMC and PMA relations, one derives the quite unrealistic large masses

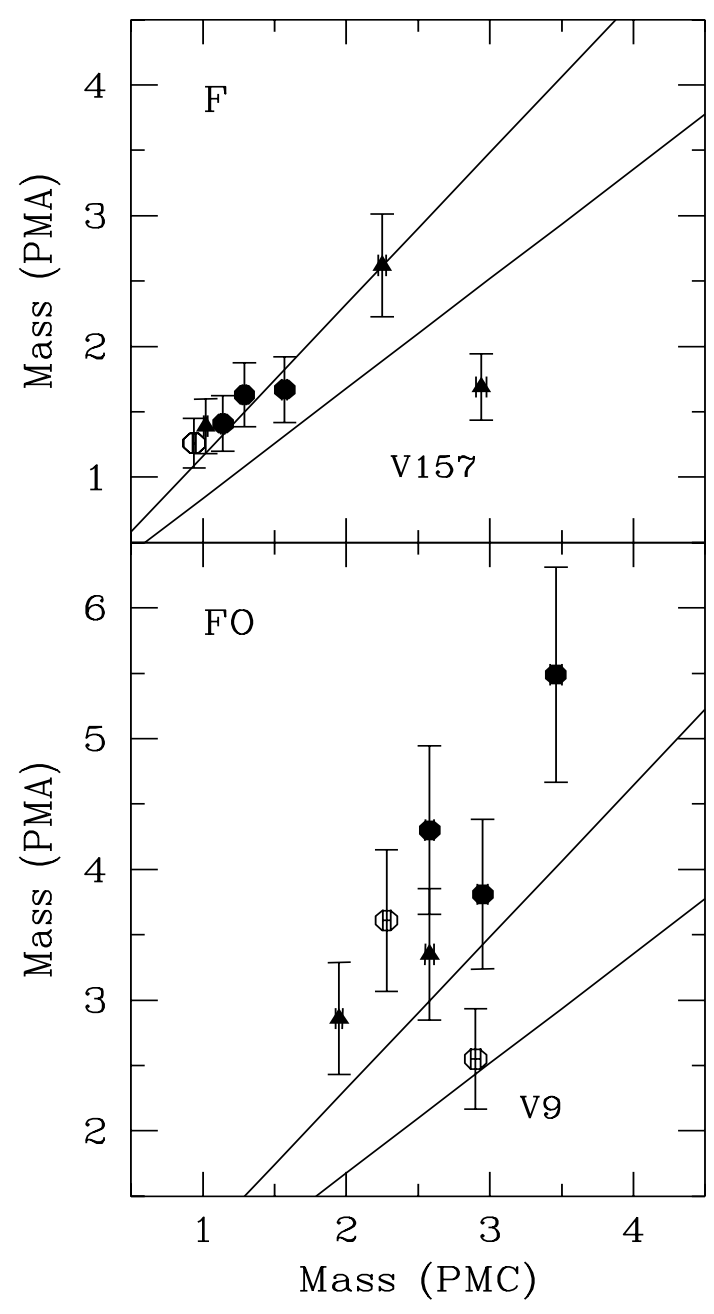

Fig. 12. (upper panel) - Comparison between the mass values of observed fundamental ACs (symbols as in Fig. 11), as inferred by the predicted PMA and PMC relations. The solid lines depict the allowed uncertainty. (lower panel) - The same, but for observed firstovertone ACs treated like fundamental pulsators.

given in brackets in Table 6. Moreover, as shown in the lower panel in Fig. 12, the difference between the two mass values is now significantly larger $(\Delta \log M \sim-0.17)$ than the allowed uncertainty, with the exception of V9 in Sextans. In summary, observed data seems to support the hypothesis that, if the variable is a fundamental pulsator, then the mass values inferred from the predicted PMC and PMA relations are internally consistent within the errors. However the location of V9 
Table 6. Estimated mass values for F and FO anomalous Cepheids, as inferred by PMC and PMA relations. The mode pulsation given in the first column is taken by previous studies, while that given in the last column is determined in the present paper (see text).

\begin{tabular}{|c|c|c|c|c|c|c|c|c|}
\hline id & $\begin{array}{c}P \\
\text { (days) }\end{array}$ & $\begin{array}{c}V \\
(\mathrm{mag})\end{array}$ & $\begin{array}{c}B \\
(\mathrm{mag})\end{array}$ & $\begin{array}{c}A_{\mathrm{V}} \\
(\mathrm{mag})\end{array}$ & $\begin{array}{c}M / M_{\odot} \\
\left(\mathrm{PMC}_{\mathrm{F}}\right)\end{array}$ & $\begin{array}{c}M / M_{\odot} \\
\left(\mathrm{PMC}_{\mathrm{FO}}\right)\end{array}$ & $\begin{array}{c}M / M_{\odot} \\
\left(\mathrm{PMA}_{\mathrm{F}}\right)\end{array}$ & Mode \\
\hline \multicolumn{9}{|l|}{ And VI } \\
\hline V06-F & 0.629 & 24.53 & 24.87 & 0.74 & 1.14 & - & 1.41 & $\mathrm{~F}$ \\
\hline V44-FO & 0.760 & 23.62 & 23.99 & 0.53 & (2.95) & 1.84 & (3.81) & FO \\
\hline V52-FO & 0.725 & 23.57 & 23.88 & 0.52 & (2.58) & 1.71 & (4.30) & FO \\
\hline V83-FO & 0.674 & 23.47 & 23.79 & 0.50 & $(3.46)$ & 2.21 & (5.49) & FO \\
\hline V84-F & 1.357 & 23.66 & 24.05 & 0.60 & 1.29 & - & 1.63 & $\mathrm{~F}$ \\
\hline V93-F & 0.477 & 24.75 & 25.13 & 0.60 & 1.57 & - & 1.67 & $\mathrm{~F}$ \\
\hline \multicolumn{9}{|l|}{ Leo II } \\
\hline V1-F & 0.408 & 21.97 & - & 0.76 & - & - & 1.46 & - \\
\hline V51-FO & 0.396 & 21.59 & - & 0.77 & - & - & - & - \\
\hline V27-F & 1.486 & 20.45 & - & 1.24 & - & - & 1.34 & - \\
\hline V203-F & 1.380 & 20.59 & - & 1.05 & - & - & 1.38 & - \\
\hline \multicolumn{9}{|l|}{ Draco } \\
\hline V55-F & 0.552 & 19.44 & - & 0.57 & - & - & 1.93 & - \\
\hline V119-F & 0.907 & 19.03 & - & 1.00 & - & - & 1.31 & - \\
\hline V134-FO & 0.592 & 18.78 & 19.06 & 0.88 & (2.58) & 1.66 & (3.35) & FO \\
\hline V141-F & 0.901 & 19.12 & 19.43 & 0.72 & 1.02 & - & 1.39 & $\mathrm{~F}$ \\
\hline V157-F & 0.936 & 18.77 & 19.24 & 1.04 & 2.94 & - & 1.69 & $\mathrm{~F}$ \\
\hline V194-F & 1.590 & 18.12 & 18.53 & 0.46 & 2.25 & - & 2.62 & $\mathrm{~F}$ \\
\hline V204-FO & 0.454 & 19.24 & 19.49 & 0.78 & (1.95) & 1.24 & (2.86) & FO \\
\hline V208-F & 0.608 & 19.28 & - & 0.33 & - & - & 2.37 & - \\
\hline \multicolumn{9}{|l|}{ Sextans } \\
\hline V9-FO & 0.293 & 19.99 & 20.32 & 0.91 & (2.90) & 1.85 & $(2.55)$ & FO \\
\hline V34-FO & 0.341 & 19.88 & 20.34 & - & - & 2.96 & - & - \\
\hline V5-F & 0.862 & 19.51 & 19.83 & 0.68 & 0.94 & - & 1.26 & $\mathrm{~F}$ \\
\hline V19-FO & 0.491 & 19.46 & 19.96 & - & - & 3.46 & - & - \\
\hline V6-F & 0.926 & 19.18 & 19.47 & - & 1.17 & - & - & - \\
\hline V1-FO & 0.693 & 18.83 & 19.08 & 0.82 & (2.28) & 1.41 & (3.61) & FO \\
\hline \multicolumn{9}{|l|}{ Sculptor } \\
\hline V5689-F & 0.855 & 19.14 & - & 0.70 & - & - & 1.55 & - \\
\hline V119-F & 1.159 & 18.86 & - & 0.55 & - & - & 1.58 & - \\
\hline V26-F & 1.346 & 18.55 & - & 0.80 & - & - & 1.67 & - \\
\hline \multicolumn{9}{|l|}{ Carina } \\
\hline V14 & 0.475 & 19.84 & 20.13 & 0.70 & 2.21 & 1.43 & 3.06 & FO \\
\hline V29 & 0.718 & 18.97 & 19.25 & 0.82 & 3.31 & 2.15 & 4.88 & FO \\
\hline V33 & 0.590 & 19.76 & 20.10 & 0.63 & 2.25 & 1.44 & 2.67 & FO \\
\hline V158 & 0.630 & 20.33 & 20.73 & 0.46 & 1.32 & 0.82 & 1.34 & $\mathrm{~F}$ \\
\hline V182 & 0.785 & 20.13 & 20.53 & 0.34 & 1.18 & 0.73 & 1.37 & $\mathrm{~F}$ \\
\hline V187 & 0.475 & 19.21 & 19.41 & 1.10 & 3.22 & 2.13 & 5.30 & FO \\
\hline V193 & 0.424 & 19.26 & 19.50 & 0.15 & 4.39 & 2.94 & 9.91 & FO \\
\hline V205 & 0.383 & 19.63 & 19.85 & 0.16 & 3.03 & 2.01 & 7.12 & FO \\
\hline
\end{tabular}

in Sextans, if this variable is a FO pulsator, would suggest that this condition is necessary but not sufficient to discriminate the fundamental mode. On this ground, according to the mass values listed in Table 6, let us adopt that V158 and V182 in Carina are bona fide fundamental pulsators, whereas V29, V187, V193 and V205 should pulsate in the first-overtone mode. As for V33 and V14, no discrimination is presently made.

Once the pulsation mode is discriminated, $\mathrm{F}$ and FO variables in Table 6 can be used to get some useful empirical relations. We show in Fig. 13 that the Period-Magnitude (PM) 


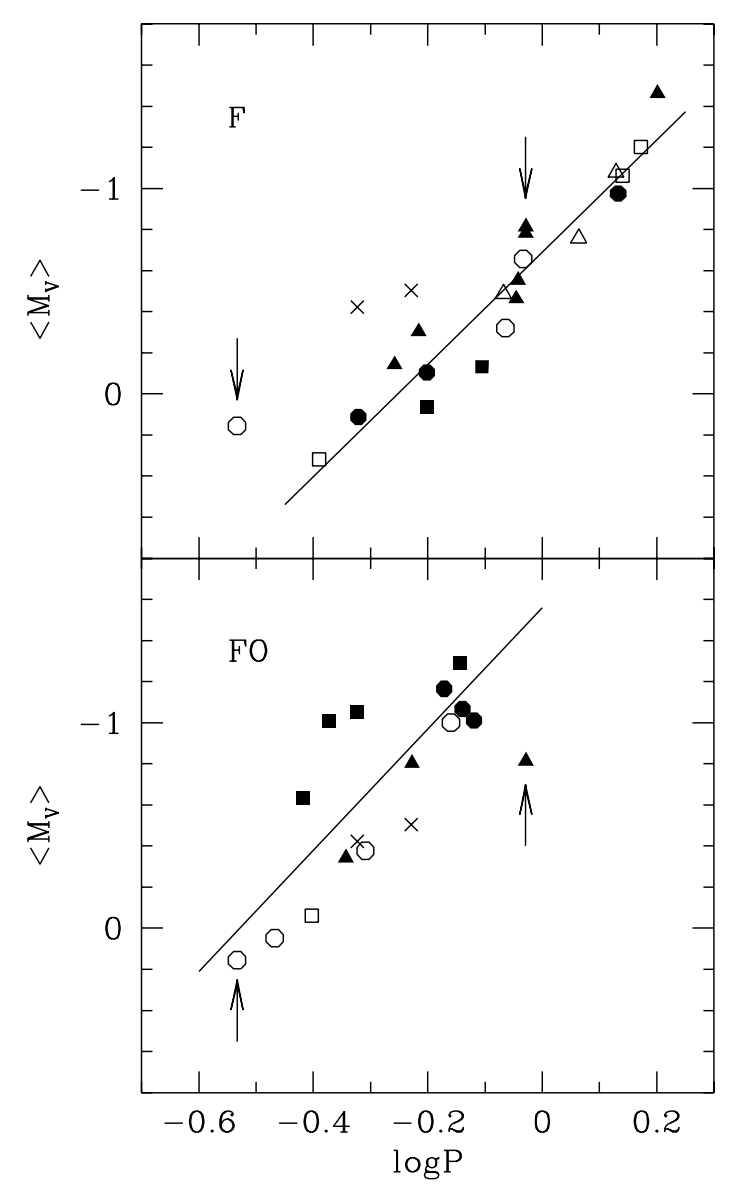

Fig. 13. Empirical $M_{V}-\log P$ relations for observed fundamental (upper panel) and first-overtone ACs (lower panel). The small arrows show V157 in Draco and V9 in Sextans discussed in the text. The solid lines are from Eqs. (9a) and (9b) in this paper.

relations for fundamental (including V158 and V182 in Carina) and first-overtone pulsators (including V29, V187 and V193 in Carina) can be linearly approximated as

$<M_{V}>_{\mathrm{F}}=-0.69( \pm 0.14)-2.73 \log P_{\mathrm{F}}$,

$<M_{V}>_{\mathrm{FO}}=-1.56( \pm 0.25)-2.95 \log P_{\mathrm{FO}}$,

which are in fair agreement with the relations recently provided by Pritzl et al. (2002).

Moreover, using only the variables with $B V$ data, we plot in Fig. 14 the absolute $\langle W B V\rangle$ quantities versus period. We derive that the $\mathrm{PW}$ relations are

$<W B V>_{\mathrm{F}}=-1.72( \pm 0.20)-3.34 \log P_{\mathrm{F}}$,

$<W B V>_{\mathrm{FO}}=-2.26( \pm 0.20)-2.44 \log P_{\mathrm{FO}}$.

Finally, using only fundamental variables, we show in Fig. 15 that the empirical fundamental PMA relation can be written as

$<M_{V}>_{\mathrm{F}}=-0.37( \pm 0.18)-2.57\left(\log P_{\mathrm{F}}+0.188 A_{\mathrm{V}}\right)$.

In these figures, the arrows depict the variables V157 in Draco (filled triangle) and V9 in Sextans (open dot), while the small crosses show V14 and V33 in Carina for which there is no clear classification. By inspection of the three figures, one derives

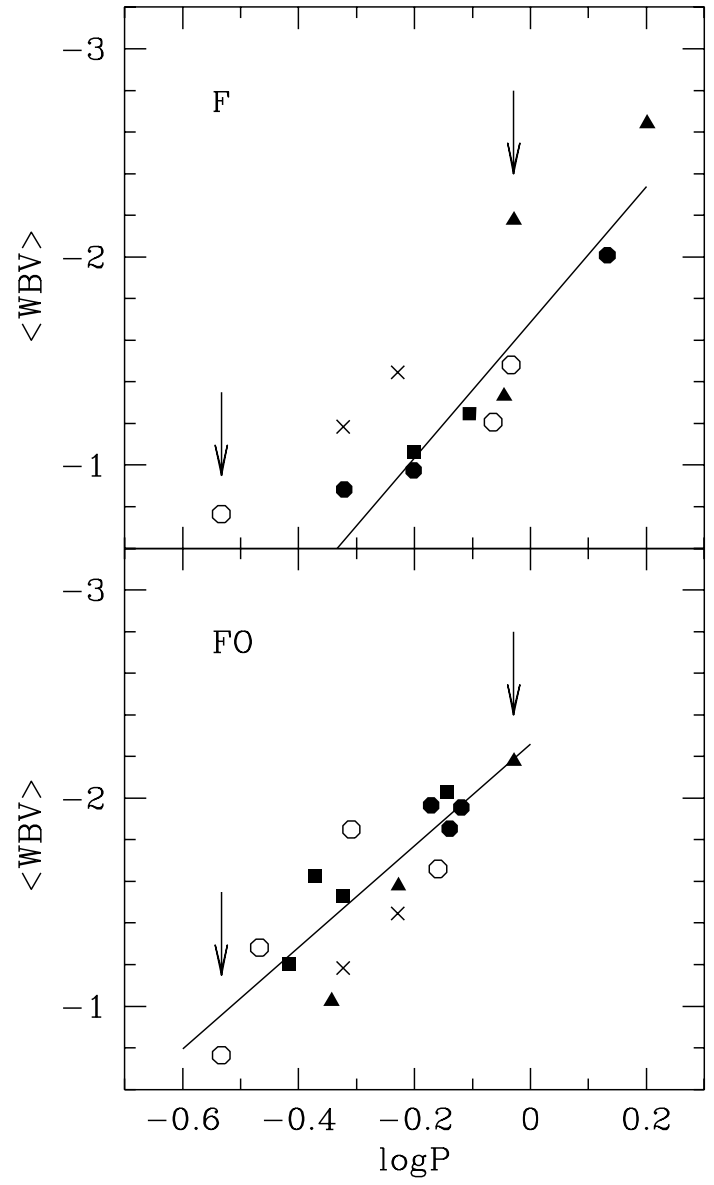

Fig. 14. As in Fig. 13, but with empirical $\langle W B V>$ quantities. The solid lines are from Eqs. (10a) and (10b) in this paper.

that V157 in Draco follows the fundamental PM and PMA relations, but the PW relation for FO pulsators, likely suggesting that its measured $B V$ color is too red. As for V9 in Sextans and the two variables in Carina, one derives that they follow more closely the FO relations rather than the fundamental ones.

In conclusion, the mode discrimination for a sample of anomalous Cepheids requires the knowledge of periods, colors and amplitudes. Assuming that the variables listed in Table 6 are representative of the average properties of ACs, fundamental or first-overtone candidates should agree with all the above empirical relations.

In order to show the importance of both color and amplitude measurements, let us take into consideration the ACs observed in Fornax by Bersier \& Wood (2002). Using the VI data listed in Table 7, the predicted PMC relations for F and FO pulsators (see Table 2) yield the mass values listed in Cols. (5) and (6), respectively. As a whole, the results are consistent with the mass values listed in Cols. (6) and (7) in Table 6, except the variable J024002.7 which appears to be too massive. Excluding this variable, we show in the upper panel of Fig. 16 the comparison between observed magnitudes, corrected with a visual distance modulus $\mu_{V}=20.79 \mathrm{mag}$ (from the distance and reddening values reported in Table 5), and the above empirical PL relations for $\mathrm{F}$ and $\mathrm{FO}$ variables. One has that most of the variables appear to be fundamental pulsators. 
Table 7. Estimated mass values anomalous Cepheids in Fornax, as inferred by PMC relations. The mode pulsation given in the last column is determined in the present paper (see text).

\begin{tabular}{|c|c|c|c|c|c|c|}
\hline id & $\begin{array}{c}P \\
\text { (days) } \\
\end{array}$ & $\begin{array}{c}V \\
(\mathrm{mag})\end{array}$ & $\begin{array}{l}V-I \\
(\mathrm{mag})\end{array}$ & $\begin{array}{c}M / M_{\odot} \\
\left(\mathrm{PMC}_{\mathrm{F}}\right)\end{array}$ & $\begin{array}{c}M / M_{\odot} \\
\left(\mathrm{PMC}_{\mathrm{FO}}\right)\end{array}$ & Mode \\
\hline J023843.0 & 1.045 & 19.80 & 0.56 & 1.91 & 1.12 & (F-FO) \\
\hline J023852.4 & 1.250 & 19.91 & 0.60 & 1.47 & 0.83 & (F) \\
\hline J024017.4 & 1.198 & 19.99 & 0.61 & 1.44 & 0.81 & $(\mathrm{~F})$ \\
\hline J023952.5 & 1.311 & 20.25 & 0.90 & 2.44 & 1.40 & (FO) \\
\hline J024002.7 & 0.533 & 20.36 & 0.85 & 7.18 & 4.96 & $?$ \\
\hline J024022.8 & 0.838 & 20.48 & 0.53 & 1.05 & 0.60 & (F) \\
\hline J023946.2 & 0.922 & 20.57 & 0.79 & 1.94 & 1.13 & (F) \\
\hline J023941.5 & 0.573 & 20.62 & 0.48 & 1.36 & 0.82 & (F) \\
\hline J023937.7 & 0.546 & 20.65 & 0.73 & 3.24 & 2.10 & (F-FO) \\
\hline J024058.3 & 0.481 & 20.79 & 0.66 & 2.70 & 1.75 & (F-FO) \\
\hline J023907.1 & 0.508 & 20.79 & 0.77 & 3.60 & 2.35 & (FO) \\
\hline J024050.2 & 0.506 & 20.83 & 0.73 & 2.97 & 1.92 & (F-FO) \\
\hline J024000.9 & 0.416 & 20.83 & 0.48 & 1.74 & 1.10 & (F-FO) \\
\hline J023926.8 & 0.504 & 20.83 & 0.67 & 2.47 & 1.58 & (F-FO) \\
\hline J024016.9 & 0.533 & 20.84 & 0.43 & 1.00 & 0.59 & $(\mathrm{~F})$ \\
\hline J023953.5 & 0.611 & 20.85 & 0.66 & 1.70 & 1.03 & (F) \\
\hline J023954.8 & 0.574 & 21.02 & 0.48 & 0.83 & 0.48 & (RR?) \\
\hline
\end{tabular}

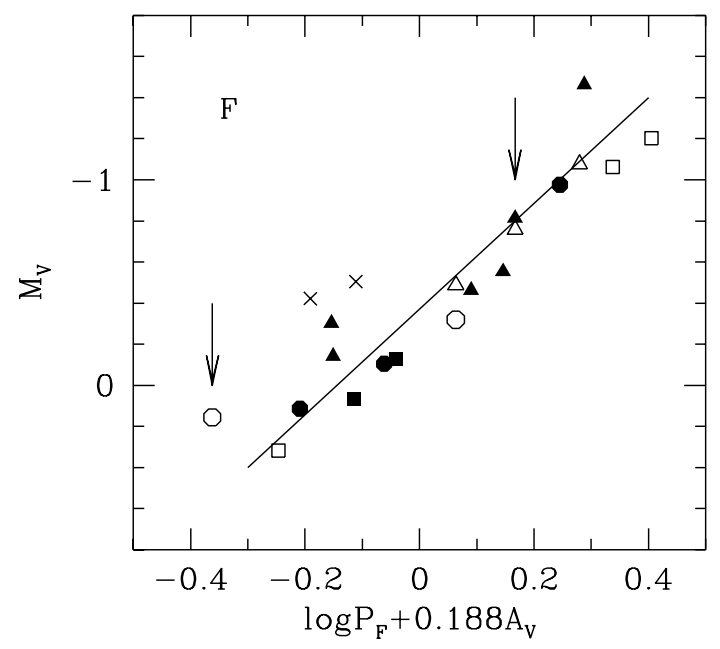

Fig. 15. Empirical PMA relation for fundamental AC. The small arrows show V157 in Draco and V9 in Sextans and the solid line is from Eq. (11) in this paper.

On the other hand, since our pulsation models give

$<W V I>=-0.22( \pm 0.001)+1.03<W B V>$

Eqs. (10a) and (10b) can be easily transformed into

$<W V I>_{\mathrm{F}}=-1.93( \pm 0.20)-3.34 \log P_{\mathrm{F}}$

$<W V I>_{\mathrm{FO}}=-2.52( \pm 0.20)-2.51 \log P_{\mathrm{FO}}$

giving us a further opportunity for determining the pulsation mode. As shown in the lower panel of Fig. 16, there are now at least six variables which fit quite well the empirical FO relation. Such a discrepancy may be due to poor light curves in the $I$ band, but without the further constrains provided by the
PMA relation, almost for half of the whole sample of variables in Fornax we are unable to clearly discriminate between $\mathrm{F}$ and FO pulsators.

\subsection{The case of Leo $A$}

Recent results of a search for short-period variable stars in the metal-poor $(Z \sim 0.0004)$ Leo A dwarf irregular galaxy are presented by Dolphin et al. (2002). These authors have found 66 variables brighter than RR Lyrae stars, noticing that there is some ambiguity as to whether these objects should be classified. Eventually, they conclude that these variables could reproduce the natural extension to low metal abundance of classical Cepheids, rather than being "anomalous" Cepheids.

We show in Fig. 17 the $M_{V}-\log P$ diagram of variables with unambiguous period and quality rating $\geq 3$, adopting an apparent distance modulus of $24.6 \mathrm{mag}$ (see Dolphin et al. 2002). Filled and open symbols refer to F and FO pulsators, respectively, as given in Dolphin et al. (2002). The comparison with the data in Fig. 11 shows that the "faint" $\left(M_{V} \geq-0.5 \mathrm{mag}\right)$ variables observed in dwarf spheroidal galaxies are absent in Leo A. This evidence would suggest that the variables in Leo A are on average more massive than ACs in dwarf spheroidal galaxies.

As a fact, the comparison with the predicted boundaries of the instability strip with $M=2.2 M_{\odot}$ (solid lines), discloses a quite reasonable agreement at the low luminosities, but the occurrence of bright $\left(M_{V} \sim-1\right.$ to $\left.-1.5 \mathrm{mag}\right)$ first overtone candidates located to the left of the predicted FOBE indicates the presence of even larger masses. In order to verify such a hypothesis, we have computed few models with $4.0 M_{\odot}$, finding that the predicted FOBE at such a mass (dotted line) fits 


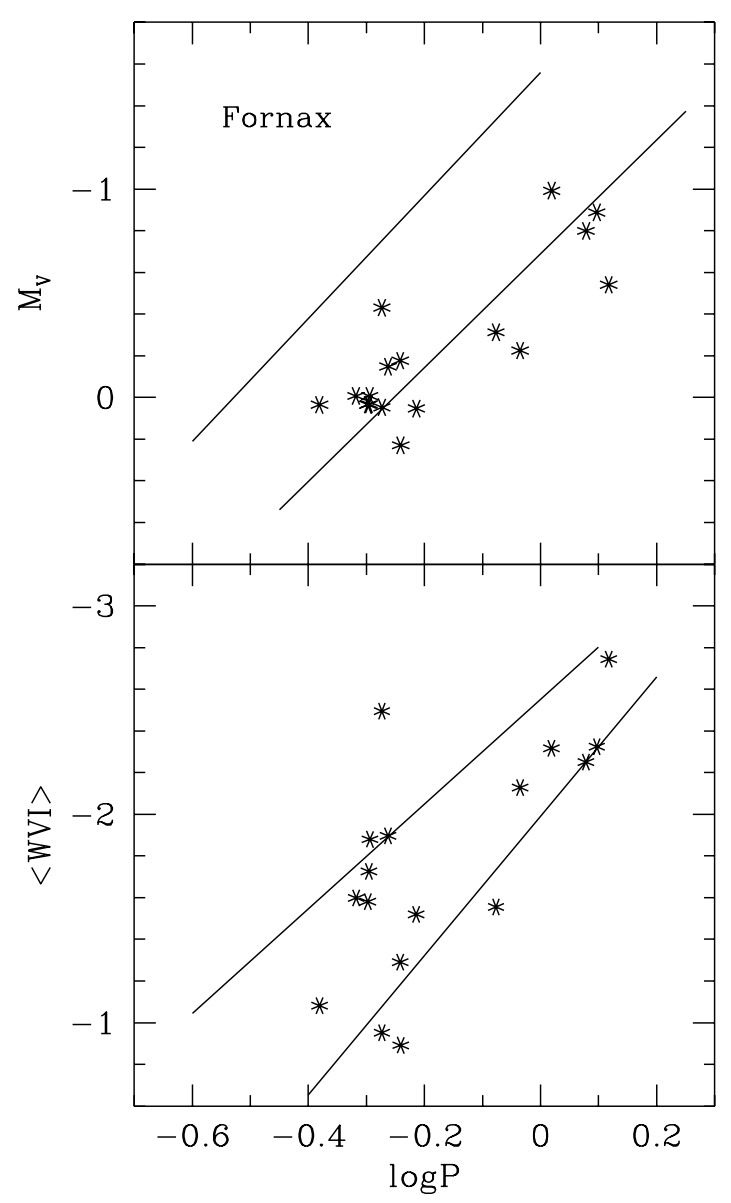

Fig. 16. (upper panel) $-M_{V}$ versus $\log P$ for ACs in Fornax. The solid lines are the same drawn in Fig. 13. (lower panel) $-\langle W V I>$ versus $\log P$ for the same variables. The solid lines are from Eqs. (10a) and (10b) in this paper.

quite well the observed distribution. Based on such a result, we believe that the variables in Leo A are a mix of "anomalous" and "classical" Cepheids. A critical analysis of these variables requires both pulsational and evolutionary models, and this issue will be addressed in a forthcoming paper (Caputo et al. in preparation).

\section{Concluding remarks}

Based on nonlinear, nonlocal and time-dependent convective pulsating models, we have presented a pulsational scenario for the analysis of "anomalous" Cepheid (AC) stars, showing that a variation of metallicity from $Z=0.0001$ to 0.0004 has a very mild effect on the edges of the instability strip, as well as on the predicted relations connecting the pulsational (period, amplitude) to the intrinsic stellar parameters (mass, luminosity, effective temperature). The main results of the present study can be summarized as follows.

1. We derive the pulsation relation connecting period with the star structural parameters $M, L, T_{\mathrm{e}}$.

2. We give the predicted relations of the boundaries of the pulsation region in the $\log L-\log T_{\mathrm{e}}$ and $M_{V}-\log P$ planes, for fixed mass. These relations, together with evolutionary

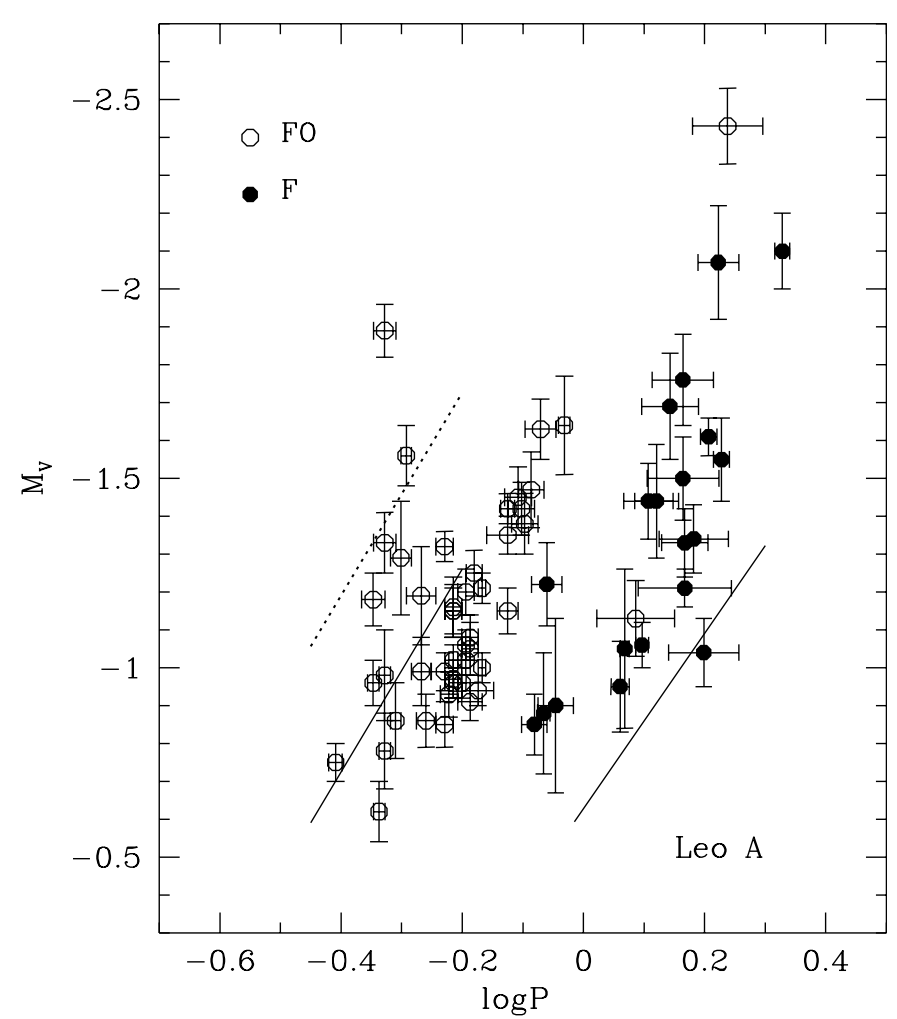

Fig. 17. $M_{V}$ versus $\log P$ for $\mathrm{F}$ (filled dots) and FO (open dots) variables in Leo A with unambiguous period and quality rating $\geq 3$, adopting an apparent distance modulus of 24.6 mag (see Dolphin et al. 2002). The solid lines represent the predicted boundaries of the instability strip with $M=2.2 M_{\odot}$, while the dotted line is the predicted FOBE at $M=4.0 M_{\odot}$ (see text for details).

models computed under different physical conditions (e.g., with or without overshooting) are the basic ingredients to evaluate whether a star is expected to evolve into the instability strip during its central He-burning phase.

3. We show that the comparison between the mean magnitudes and colors, as based on theoretical light curves transformed into the observational bands, and the corresponding static values, follows the general trend also shown by other classes of pulsating stars (RR Lyrae, classical Cepheids).

4. We derive the predicted Period-Magnitude-Color (PMC) relation, showing that it can be used to estimate, with a formal uncertainty of $\sim 2 \%$, the mass range spanned by a sample of well-measured variables at the same distance and with the same reddening. If the distance and reddening are independently known, then the mass absolute values can be determined too.

5. As usually adopted for classical Cepheids, an important tool to derive absolute distances is given by the theoretical reddening-free Wesenheit functions. These relations (PW) can also be used to get the mass range of a sample of ACs at the same distance with a formal accuracy of $\sim 5 \%$.

6. As already known for RR Lyrae stars, the amplitude of fundamental pulsators is linearly dependent on the period (logarithmic scale), for fixed mass and luminosity, providing a mass-dependent Period-Magnitude-Amplitude (PMA) relation which again can be adopted to estimate the 
mass range spanned by fundamental variables at the same distance, but with a larger formal uncertainty $(\sim 15 \%)$ with respect to the other methods.

7. Since the PMA relation only holds for fundamental pulsators, whereas PMC and PW relations can be applied both to the fundamental and the first overtone mode, the comparison between the masses evaluated by adopting the different kinds of relations may help to attempt a discrimination of the pulsation mode. Moreover, we show that the difference between the mass values inferred from PMC and PMA relations is independent of errors on the distance modulus, whereas the mass absolute values should vary by about $\pm 12 \%$ with $\delta \mu= \pm 0.1 \mathrm{mag}$.

The comparison between the predicted edges of the instability strip and the observed distribution of ACs belonging to a number of dwarf spheroidal galaxies seems to support the predictive capabilities of pulsation models. The only exception is found for few first overtone variables in the irregular galaxy Leo A, that fall to the left of the predicted blue boundary reproducing the other variables, suggesting a mass of the order of $4 M_{\odot}$.

By considering dwarf spheroidal galaxies for which a mode discrimination of ACs is available in the literature and by applying the predicted PMC and PMA relations to these variables, we are able to conclude that if the variable is a fundamental pulsator, then both PMC and PMA yield mass values which are internally consistent within the errors. On this basis, we find that only two variables in Carina are bona fide fundamental pulsators, whereas the remaining variables should pulsate in the first-overtone mode.

On the basis of the quoted mode discrimination empirical PL and PW relations for fundamental and first overtone ACs in dwarf galaxies, as well as the PMA relation for fundamental variables, are derived.

The intrinsic errors of the results listed above are valid in the context of the adopted treatment for the convective transfer. Indeed, even if our formalism has the advantages of being nonlocal and time dependent, nevertheless it has turned out recently that the inclusion of additional equations in the Reynolds momentum equations (see, e.g., Canuto \& Dubovikov 1998) improves the treatment of turbulence.

Acknowledgements. We thank our anonymous referee for his/her helpful comments and corrections that have considerably improved the manuscript. We also thank G. Bono and V. Castellani for useful suggestions and remarks. Model computations made use of resources granted by the "Consorzio di Ricerca del Gran Sasso", according to Project 6 "Calcolo Evoluto e sue Applicazioni (RSV6) Cluster C11/B". This work was financially supported by MIUR/Cofin 2002, under the project "Stellar Populations in Local Group Galaxies" (Monica Tosi coordinator) and MIUR/Cofin 2001, under the project "Origin and evolution of stellar populations in the Galactic Spheroid (Raffaele Gratton coordinator).

\section{References}

Alexander, D. R., \& Ferguson, J. W. 1994, ApJ, 437, 879

Baldacci, L., Matonti, F., Rizzi, L., et al. 2003 [astro-ph/03005506]

Bersier, D., \& Wood, P. R. 2002, AJ, 123, 840

Bono, G., \& Stellingwerf, R. F. 1994, ApJS, 93, 233B

Bono, G., Caputo, F., \& Stellingwerf, R. F. 1995, ApJS, 99, 263

Bono, G., Caputo, F., Santolamazza, P., Cassisi, S., \& Piersimoni, A. 1997a, AJ, 113, 2209 [B97]

Bono, G., Caputo, F., Castellani, V., \& Marconi, M. 1997b, A\&AS, 121, 327

Bono, G., Caputo, F., Castellani, V., \& Marconi, M. 1999a, ApJ, 512, 711

Bono, G., Marconi, M., \& Stellingwerf, R. F. 1999b, ApJS, 122, 167B

Bono, G., Castellani, V., \& Marconi, M. 2000a, ApJ, 532, 129

Bono, G., Castellani, V., \& Marconi, M. 2000b, ApJ, 529, 293

Bono, G., Castellani, V., \& Marconi, M. 2002, ApJ, 565, L83

Bono, G., Caputo, F., Castellani, V., et al. 2003, MNRAS, 344, 1097

Canuto, V. M., \& Dubovikov, M. 1998, ApJ, 493, 834

Caputo, F. 1998, A\&ARv, 9, 33C

Caputo, F., Castellani, V., Marconi, M., \& Ripepi, V. 2000, MNRAS, 316,819

Caputo, F., Marconi, M., \& Musella, I. 2000, A\&A, 354, 610

Caputo, F., Marconi, M., Musella, I., \& Pont, F. 2001, A\&A, 372, 544

Caputo, F., Marconi, M., Musella, I., \& Santolamazza, P. 2000, A\&A, 359,1059

Caputo, F., Marconi, M., \& Ripepi, V. 1999, ApJ, 525, 784

Cardelli, J. A., Clayton, G. C., \& Mathis, J. S. 1989, ApJ, 345, 245

Castellani, V., \& Degl'Innocenti, S. 1995, A\&A, 298, 827

Castelli, F., Gratton, R. G., \& Kurucz, R. L. 1997a, A\&A, 318, 841

Castelli, F., Gratton, R. G., \& Kurucz, R. L. 1997b, A\&A, 324, 432

Dall'Ora, M., Ripepi, V., Caputo, F., et al. 2003, AJ, 126, 197

Dolphin, A., Saha, A., Claver, J., et al. 2002, AJ, 123, 3154

Fiorentino, G., Caputo, F., Marconi, M., \& Musella, I. 2002, ApJ, 576, 402

Girardi, L., Bressan, A., Bertelli, G., \& Chiosi, C. 2000, A\&AS, 141, $371 \mathrm{G}$

Iglesias, C. A., \& Rogers, F. J. 1996, ApJ, 464, 943

Kaluzny, J., Kubiak, M., Szymanski, M., et al. 1995, A\&A, 112, 407

Madore, B. F. 1982, APJ, 253, 575

Madore, B. F., \& Freedman, W. L. 1991, PASP, 103, 933

Marconi, M., Caputo, F., Di Criscienzo, M., \& Castellani, M. 2003, ApJ, 596, 299

Mateo, M. 1998, ARA\&A, 36, 435

Mateo, M., Fisher, P., \& Krzeminski, W. 1995, AJ, 110, 2166

Nemec, J. M., Nemec, A. F. L., \& Lutz, T. E. 1994, AJ, 108, 222 [NNL]

Nemec, J. M., Wehlau, A., \& Mendes de Oliveira, C. 1988, AJ, 96, 528

Pritzl, B. J., Armandroff, T. E., Jacoby, G. H., \& Da Costa, G. S. 2002, AJ, 124, 1464

Sandage, A. 1958, ApJ, 127, 513

Tanvir, N. R. 1999, in Post-Hipparcos Cosmic Candles, ed. A. Heck, \& F. Caputo (Dordrecht: Kluwer Academic Publishers), 17

van Albada, T. S., \& Baker, N. 1971, ApJ, 169, 311

van Albada, T. S., \& Baker, N. 1973, ApJ, 185, 477

Wallerstein, G. 2002, PASP, 114, 689 